\title{
Dynamical response of a pinned two-dimensional Wigner crystal
}

\author{
Michael M. Fogler \\ School of Natural Sciences, Institute for Advanced Study, Einstein Drive, Princeton, New Jersey 08540 \\ David A. Huse \\ Physics Department, Princeton University, Princeton, New Jersey 08544
}

\begin{abstract}
We re-examine a long-standing problem of a finite-frequency conductivity of a weakly pinned two-dimensional classical Wigner crystal. In this system an inhomogeneously broadened absorption line (pinning mode) centered at disorder and magnetic field dependent frequency $\omega_{p}$ is known to appear. We show that the relative linewidth $\Delta \omega_{p} / \omega_{p}$ of the pinning mode is of the order of one in weak magnetic fields, exhibits a power-law decrease in intermediate fields, and eventually saturates at a small value in strong magnetic fields. The linewidth narrowing is due to a peculiar mechanism of mixing between the stiffer longitudinal and the softer transverse components of the collective excitations. The width of the high-field resonance proves to be related to the density of states in the low-frequency tail of the zero-field phonon spectrum. We find a qualitative agreement with recent experiments and point out differences from the previous theoretical work on the subject.
\end{abstract}

\section{INTRODUCTION}

The problem of pinning of an elastic solid by an external random potential has been a long-standing problem of condensed matter physics, first addressed by Larkint in 1970. Physical systems where this problem arises include charge-density waves 3 vortex lattifes in superconductors, 3 interfaces 1 magnetic bubbles, 3 liquid crystals in aerogels 1 and many others. An interesting example of the charge-density wave is a Wigner crystal (WC). Under terrestrial conditions a stable WC has been realized in its two-dimensional (2D) form, 8 on cryogenic surfaces and in semiconductor heterostructures with low carrier density and/or in strong magnetic fields. In this work we examine the finite-frequency response of a collectively pinned $2 \mathrm{D}$ WC. The collective pinning is the regime where individual defects of the substrate are too weak to significantly deform the crystalline lattice, so that the WC is well ordered ("unpinned") at small length scales, but the cumulative effect of the disorder eventually dominates the elasticity at a pinning length, which is much larger than the lattice constant.

The linear-response conductivity $\sigma_{\alpha \beta}(q, \omega)$ of the WC in an external magnetic field is a tensor. We focus our study on the real part of its diagonal component $\sigma_{x x}(q=0, \omega)$, the quantity that determines the power absorption in the presence of a uniform electric field. If the frequency $\omega$ is not too small, the absorption is dominated by the collective excitations. In the absence of the magnetic field and random potential, a 2D WC possesses two branches of such excitations: the longitudinal (L) and the transverse $(\mathrm{T})$ phonons. The long-wavelength L-phonon is also referred to as plasmon. In a finite magnetic field L- and T-phonons hybridize into the magnetophonons (gapless lower hybrid mode) and the magnetoplasmons (gapped higher hybrid mode). It is noteworthy that without the pinning potential, the frequency and the oscillator strength of the magnetophonons vanish at $q=0$ by virtue of Kohn's theorem. The absorption of a spacially uniform ac field is possible only by exciting the magnetoplasmon mode, at the cyclotron frequency $\omega_{c}=e B / m_{e} c$ set by the magnetic field $B$. The pinning shifts the original $q=0$ magnetophonon mode to a nonzero disorder-dependent frequency $\omega_{p}$, broadens it, and imparts it with a finite oscillator strength. The resulting absorption line, which can be called the pinning made, was first discussed by Fukuyama and Lee (FL). 10 In those early works the linewidth $\Delta \omega_{p}$ of the pinning mode was predicted to be of the order of $\omega_{p}$ itself. Strictly speaking, FL considered not a WC but a more conventional charge-density wave where the charge modulation has only one harmonic, i.e., is cosine-like. Experiments on such materials, e.g., $\mathrm{K}_{0.3} \mathrm{MoO}_{3}$ ("blue bronze"), $\mathrm{TaS}_{3}$, etc., performed in zero magnetic field indeed revealed broad absorption lines at disorder-dependent frequencies.11 A later work of Normand et al. 12 devoted specifically to the WC in a strong $B$ revised some results of FL but left unchanged the prediction that $\Delta \omega_{p} \widetilde{\sim} \omega_{p}$. At the time, experiments seemed to confirm that. 13 It came as a surprise when most recent measurements in strong magnetic fields 417 demonstrated that $\Delta \omega_{p}$ can be more than order of magnitude smaller than $\omega_{p}$. Such unexpected findings revived interest in this long-standing problem.

An important step toyards resolution of the puzzle has been made by Fertig, 18 who showed that long-range Coulomb interaction plays an important role in reducing the inhomogeneous broadening of the pinning mode. However, Fertig used an oversimplified model (commensurate pinning) and did not calculate $\Delta \omega_{p}$ directly. His results for another quantity may be interpreted as an indication that $\Delta \omega_{p} / \omega_{p}$ is exponentially small for weak pip ning. More realistic model was studied by Chitra et al. $\mathrm{t}$ who considered pinning by a short-range Gaussian ran- 
dom potential of a general type. We comment on their results later in this section.

In the present paper we study essentially the same model as FL and Chitra et al. except we treat the electrons classically. This limits the applicability of our results to the case $\xi>l_{B}$, where $\xi$ is the correlation length of the pinning potential and $l_{B}=\sqrt{\hbar c / e B}$ is the magnetic length. The classical approximation enables us to focus on the interaction of the WC with disorder, which is really the essence of the pinning mode phenomenon. We critically re-examine the previous work on the subject, identify the physical mechanism responsible for the line narrowing, and spell out the conditions under which it occurs:

- the lattice dynamics is inertial (not overdamped)

- magnetic field is sufficiently strong, such that $\omega_{c}$ is larger than $\omega_{p}$

- the pinning is sufficiently weak so that $\omega_{p}$ is much smaller than the magnetophonon bandwidth

- the compression modulus $\lambda$ of the WC evaluated at the pinning length is larger than its shear modulus $\mu$ (satisfied for long-range interaction).

If any of the conditions above is violated, then the line is conventionally broad, $\Delta \omega_{p} \sim \omega_{p}$.

Although we were unable to find the exact expression for $\Delta \omega_{p}$, we show that there is an asymptotically exact relation between $\Delta \omega_{p}$ at intermediate $B$ and the lowfrequency tail of the phonon spectral function in zero $B$ (we remind the reader here that we study classical electrons, which form the WC at any $B$ ). Establishing such a connection and elucidating the physical mechanism of the line narrowing are our main achievements.

The phonon spectral function at low frequencies is expected to have a power law form

$$
\operatorname{Im} D_{\alpha \alpha}(q=0, \omega) \propto \omega^{2 s+1},
$$

with a nontrivial exponent $s$. This additional input and further considerations enable us to predict that as $\omega_{c}$ increases and becomes larger than $\omega_{p}, \Delta \omega_{p} / \omega_{p}$ first decreases in a power-law fashion

$$
\Delta \omega_{p} / \omega_{p} \sim\left(\omega_{p} / \omega_{c}\right)^{s}
$$

but then eventually saturates at a value (see Fig. 11)

$$
\Delta \omega_{p} / \omega_{p} \sim(\mu / \lambda)^{s}
$$

(here $\lambda$ is meant to be evaluated at the pinning length). The recent experiments 1417 have probed the high-field regime where Eq. (3) is supposed to apply.

In the aforementioned work of Chitra et al.19 a set of equations was derived, which, when analyzed further, also yield Eq. (3), with $s=\frac{1}{2}$. This specific value of $s$ can be traced down to the fact that Chitra et al. unknowingly rederive a common form of the self-consistent
Born approximation (SCBA), where Eq. (1) with $s=\frac{1}{2}$ is satisfied. Since the SCBA is uncontrolled at small $\omega$, these results are unreliable. Moreoyer, appealing to the known properties of Lifshitz tails 20 in other disordered systems, we argue that the SCBA strongly overestimates the low-frequency spectral function. We propose that the correct approach should be based on considering certain low-propability disorder configurations ("optimal fluctuations"), which leads to

$$
s=\frac{3}{2} \text {. }
$$

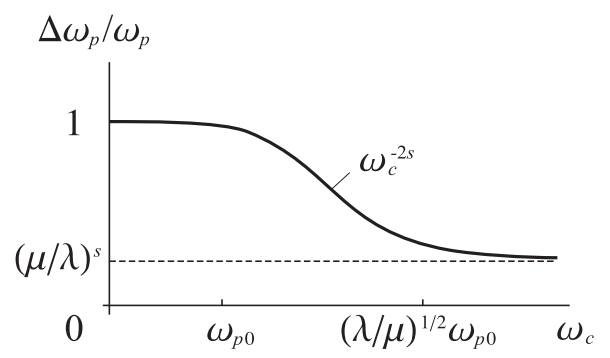

FIG. 1. Relative linewidth of the pinning mode as a function of the cyclotron frequency.

The paper is organized as follows. In Sec. II we discuss the ground state of the pinned WC, placing emphasis on the parameters that provide the input for the dynamical problem of interest. In Sec. III] we formulate the model and the general framework for study of the finitefrequency response. In Sec. IV] we derive Eq. (2). In Sec. $\mathrm{Y}$ we study the soft phonon modes at $B=0$ and obtain the above formula for $s$. The comparison with the experiments is given in Sec. VII after a brief discussion of quantum and finite-temperature effects in Sec. VI.

\section{STATIC PROPERTIES}

The distortions of the lattice in the ground state of a collectively pinned Wigner crystal accumulate gradually over length scales much larger than the lattice constant a. Such distortions can be described in terms of a smooth displacement field $\mathbf{u}^{(0)}(\mathbf{r})$. The Hamiltonian of the system $H=H_{e l}+H_{p}$ is the sum of the elastic term,

$$
\begin{gathered}
H_{e l}=\frac{1}{2} \int_{\mathbf{q}} u_{\alpha}^{(0)}(-\mathbf{q}) H_{\alpha \beta}^{0}(\mathbf{q}) u_{\beta}^{(0)}(\mathbf{q}), \\
H_{\alpha \beta}^{0}=\left(\delta_{\alpha \beta}-\frac{q_{\alpha} q_{\beta}}{q^{2}}\right) H_{T}^{0}(q)+\frac{q_{\alpha} q_{\beta}}{q^{2}} H_{L}^{0}(q), \\
H_{T}^{0}(q)=\mu q^{2}, \quad H_{L}^{0}(q)=\lambda(q) q^{2},
\end{gathered}
$$

and the pinning term,21

$$
\begin{gathered}
H_{p}=n_{e} \int_{\mathbf{r}} U(\mathbf{r})\left\{\sum_{\mathbf{K}} \cos \mathbf{K}\left[\mathbf{r}-\mathbf{u}^{(0)}(\mathbf{r})\right]-\nabla \mathbf{u}^{(0)}\right\}, \\
\langle U(\mathbf{r}) U(0)\rangle=C(r) .
\end{gathered}
$$


The notations here are as follows. The q-integral is taken over the Brillouin zone with measure $d^{2} q /(2 \pi)^{2}$; the $\mathbf{r}$-integrals is taken over the area of the system with measure $d^{2} r$. Parameters $\mu$ and $\lambda$ represent the shear and bulk elastic moduli of the WC, respectively. In strong magnetic fields, i.e., at low filling factors they approach their classical values $22 \mu \approx 0.245 e^{2} n_{e}^{3 / 2} / \kappa$ and $\lambda(q)=-5 \mu+Y /|q|$. Here $n_{e}=2 / \sqrt{3} a^{2}$ is the average electron concentration, $\kappa$ is the dielectric constant of the medium, and $Y=2 \pi e^{2} n_{e}^{2} / \kappa$. The infrared divergence of $\lambda(q)$ originates from the long-range Coulomb interaction. K's are the reciprocal lattice vectors. Finally, the correlator $C(r)$ is assumed to rapidly decay at $r>\xi$ where $\xi<a$ is the correlation length of the short-range Gaussian random potential $U(\mathbf{r})$.

The static properties of the system defined by Eqs. (5 9) can be investigated using classical statistical mechanics methods. Problems of this type have been studied extensively in the past, see Ref. 3 for review and references, and also some recent contributions, Refs. 21, 23 26. The $2 \mathrm{D}$ WC generally conforms to the $d=2, m=2$ class of elastic media ( $d$ is the total number of spacial dimensions and $m$ is the number of components of $\mathbf{u}$ ). However, some caution is required in transferring the known results to the $\mathrm{WC}$ case because the $\mathrm{L}$ - and $\mathrm{T}$-components are not equivalent. The large disparity $\lambda \gg \mu$ of the elastic moduli causes strongly suppression of the longitudinal distortions compared to the transverse ones. Therefore, in certain formulas one has to use $m=1$. In this respect, the WC is similar to the Abrikosov vorter lattice where the analogous inequality $c_{11} \gg c_{66}$ exists.

Let us define the two-point correlation function (roughness) $W(r)=\left\langle\left[\mathbf{u}^{(0)}(\mathbf{r})-\mathbf{u}^{(0)}(0)\right]^{2}\right\rangle^{1 / 2}$. Many static properties of the system can be deduced from the behavior of $W(r)$. Three regimes can be distinguished: randomforce, random-manifold, and the asympotic (Fig. 2).

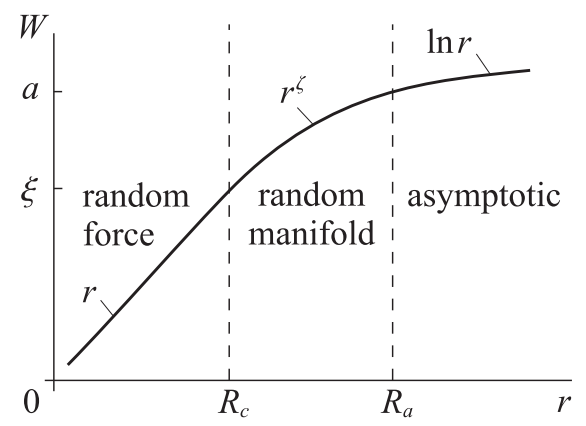

FIG. 2. Roughness $W(r)$.

The random-force or Larkin regime appears for small $r$ where $W(r)<\xi$. The last inequality legitimizes expanding the exponential in Eq. (8) and keeping only the linear in $u^{(0)}$ terms. The Hamiltonian becomes quadratic, and its ground state is easily found to be

$$
u_{\alpha}^{(0)}(\mathbf{q})=-i n_{e} \sum_{\mathbf{K}}\left[\mathbf{H}^{0}\right]_{\alpha \beta}^{-1}(\mathbf{q}) K_{\beta} \tilde{U}(\mathbf{q}+\mathbf{K}) .
$$

Here and below tildes denote Fourier transforms.

Averaging over the disorder, we arrive at

$$
\begin{aligned}
W^{2}(\mathbf{r}) & \simeq V^{\prime}(0) \int_{\mathbf{q}}\left(1-e^{i \mathbf{q r}}\right)\left[\left(H_{T}^{0}\right)^{-2}+\left(H_{L}^{0}\right)^{-2}\right] \\
& \propto r^{2} \ln \left(R_{c} / r\right) \\
V(z) & \equiv-\frac{n_{e}^{2}}{2} \sum_{\mathbf{K}} \tilde{C}(K) e^{-z K^{2} / 2} .
\end{aligned}
$$

To obtain Eq. (12) we cut off the log-divergent integral in Eq. (11) at $q=q_{c}$ where $q_{c}=\pi / R_{c}$ and $R_{c}$ is the Larkin length defined by the relation $W\left(R_{c}\right)=\xi$. For a weak random potential $R_{c}$ is much larger than $a$ and is given by

$$
R_{c} \sim \mu a \xi^{2}[C(0)]^{-1 / 2} .
$$

The same estimate for $R_{c}$ can be obtained in a simpler way, 310 by equating the elastic energy density $\mu \xi^{2} / R_{c}^{2}$ to the pinning energy density $[C(0)]^{1 / 2} \sqrt{N} / R_{c}^{2}$. In the last expression $N=n_{e} R_{c}^{2}$ is the number of electrons within the area $R_{c} \times R_{c}$ and the square root takes into account that individual pinning energies have random signs.

The elastic-manifold regime is realized at intermediate distances, $R_{c}<r<R_{a}$ where $\xi<W(r)<a$. Here the roughness grows slower than in the Larkin regime, $W(r) \propto r^{\zeta}, \zeta<1$. For the 2D Wigner crystal the Flory estimate $\zeta=\frac{1}{3}$ (as appropriate for the $d=2$ m $m=2$ case) should be accurate but perhaps not exact 3 The slower growth rate of $W(r)$ is due to a less efficient mechanism by which the lattice adjusts to the random potential on length scales exceeding $R_{c}$. The steepest descent towards the nearest local minima of the potential landscape in the Larkin regime gets replaced by selection among a set of many possible yet roughly equivalent nearby minima.

The third and the last regime begins when $W(r)$ reaches $a$ at the length scale $R_{a}=R_{c}(a / \xi)^{1 / \zeta}$. The accumulation of the pinning energy density at such large length scales becomes additionally suppressed by lattice periodicity effects. Indeed, a uniform shift $u \rightarrow u+a$ reduces to relabeling the lattice sites with no physical significance. As a result, the roughness grows only logarithmically with distance, $W(r) \sim a \ln \left(r / R_{a}\right)$. The asymptotic $\ln r$-roughness corresponds to a "quasi-short-range" order: $W(r)$ increases so slowly that it is barely enough to remove the algebraic divergencies (Bragg peaks) of the static structure factor

$$
F(\mathbf{q})=n_{e}^{2} \int_{\mathbf{r}} \exp \left[i(\mathbf{q}-\mathbf{K}) \mathbf{r}-\frac{1}{2} K^{2} W^{2}(r)\right]
$$

at the reciprocal lattice vectors $\mathbf{q}=\mathbf{K}$. Remarkably, it also creates enough elastic stress to generate unbound dislocations, on the length scale $22 \xi_{D} \sim$ $R_{a} \exp \left[C_{0} \ln ^{1 / 2}\left(R_{a} / a\right)\right]$. At this scale $\mathbf{u}$ becomes multivalued and Eq. (5) must be modified. 
Although very interesting, the random-manifold and the asymptotic regimes do not play much role in the further discussion. We mentioned them here for the sake of a more comprehensive introduction and also to draw attention to the difference between two characteristic length scales, $R_{c}$ and $R_{a}$. $R_{a}$ is the length that seems to be important only for rather subtle properties mentioned above: the destruction of the Bragg peaks and the appearance of the topological defects. In contrast, $R_{c}$ determines most of the physically important response characteristics such as the threshold electric field in the dg transport and the frequency $\omega_{p}$ of the pinning mode. 19 This role of $R_{c}$ originates from the fact that it is the length scale where the dominant contribution $\sim \mu \xi^{2} / R_{c}^{2}$ to the pinning energy density is accumulated. In this sense $R_{c}$ plays the role of the pinning length mentioned in Sec. If.

Let us now elaborate on the difference between the Tand L-correlation functions mentioned above. To do so we decompose the elastic displacement into its $\mathrm{T}$ - and L-components,

$$
\mathbf{u}(\mathbf{q})=\hat{\mathbf{q}} u_{L}(\mathbf{q})+[\hat{\mathbf{z}} \times \hat{\mathbf{q}}] u_{T}(\mathbf{q})
$$

where $\hat{\mathbf{q}}=\mathbf{q} /|\mathbf{q}|$, and define the two components of roughness $W_{\alpha}=\left\langle u_{\alpha}^{(0)}(\mathbf{r})-u_{\alpha}^{(0)}(0)\right\rangle^{1 / 2}, \alpha=L, T$. First of all, let us examine the short-distance behavior using Eq. (10). For $W_{L}(r)$ we obtain the expression similar to Eq. (11):

$$
W_{L}^{2}(r) \simeq V^{\prime}(0) \int_{\mathbf{q}} \frac{1-e^{i \mathbf{q r}}}{\left[H_{L}^{0}(q)\right]^{2}} \simeq \frac{V^{\prime}(0)}{2 \pi Y^{2}} \ln \left(\frac{r}{a}\right)
$$

and so $W_{L}(r)$ is much smaller than $W_{T}(r) \simeq W(r)$ given by Eq. (12). Formula (17) is certainly valid for $r<R_{c}$. For larger $r$ we can no longer treat the elastic distortion $\left|\mathbf{u}^{(0)}(\mathbf{r})-\mathbf{u}^{(0)}(0)\right|$ as small. Nonetheless, we expect that the region of validity of Eq. (17) extends somewhat beyond $r=R_{c}$. Indeed, instead of doing the perturbation theory around the ideal lattice state, we can do it around the ground state $\mathbf{u}_{\infty}^{(0)}$ of the incompressible crystal, $\lambda=\infty$. In the second approach we have to account for a finite pinning energy density, but as long as the longitudinal elastic stiffness $Y q$ where $q \sim \pi / r$ exceeds the characteristic curvature $\mu q_{c}^{2}$ of the pinning energy density landscape, i.e., for $r \lesssim R_{L}=(Y / \mu) R_{c}^{2} \sim R_{c}^{2} / a$, this should be unimportant. Substituting $r=R_{L}$ into Eq. (17) we find that $W_{L}\left(R_{L}\right) \ll \xi$, contrary to some previous suggestions 12 that $W_{L}\left(R_{L}\right) \sim W_{T}\left(R_{L}\right)$ [recall that $\left.W_{T}\left(R_{L}\right) \simeq W\left(R_{L}\right) \gg W\left(R_{c}\right)=\xi\right]$.

The large-distance $\left(r>R_{L}\right)$ behavior of $W_{L}(r)$ is unaccessible within the perturbation theory. However, we should get qualitatively correct results by sian variational replica method (GVRM).21 Assuming for simplicity that $R_{L} \gg R_{a}$ and suitably modifying GVRM for the present problem, we find

$$
W_{L}^{2}(r) \propto V^{\prime}(0) \int_{\mathbf{q}} \frac{1-\cos (\mathbf{q r})}{H_{L}^{0}(q)\left[H_{L}^{0}(q)+\Pi_{a}\right]}
$$

where $\Pi_{a}=\mu R_{a}^{-2}$. It is easy to see that $W_{L}(r)$ tends to a finite limiting value as $r \rightarrow \infty$. So, the long-range positional order is absent in the $\mathrm{T}$ - but is preserved in the L-part of the elastic displacement field.27

To conclude the review of the statics, let us consider a matrix of the second derivatives of $H_{p}$,

$$
\mathbf{S}(\mathbf{r})=\frac{\partial^{2} H_{p}}{\partial \mathbf{u}^{(0)} \partial \mathbf{u}^{(0)}}=n_{e} \sum_{\mathbf{K}} \mathbf{K} \mathbf{K} U(\mathbf{r}) \cos \mathbf{K}\left[\mathbf{r}-\mathbf{u}^{(0)}(\mathbf{r})\right]
$$

Matrix $\mathbf{S}$ plays an important role in the dynamics and we need to understand its properties.

In the case of a short-range disorder we study here, the correlations in $U(\mathbf{r})$ decay more rapidly with $r$ than those of $\mathbf{u}^{(0)}(\mathbf{r})$. This enables us to find the two-point correlator of $S_{\alpha \beta}$ with little effort. It suffices to use the approximation $\mathbf{u}^{(0)}(\mathbf{r})=0$ in Eq. (19), which leads to

$$
\begin{aligned}
& \left\langle\tilde{S}_{\alpha \mu}\left(\mathbf{k}_{1}\right) \tilde{S}_{\nu \beta}\left(\mathbf{k}_{2}\right)\right\rangle-\left\langle\tilde{S}_{\alpha \mu}\right\rangle\left\langle\tilde{S}_{\nu \beta}\right\rangle \simeq-\frac{V^{\prime \prime}(0)}{2} \\
& \quad \times\left(\delta_{\alpha \mu} \delta_{\nu \beta}+\delta_{\alpha \beta} \delta_{\mu \nu}+\delta_{\alpha \nu} \delta_{\mu \beta}\right) \delta_{\mathbf{k}_{1} \mathbf{k}_{2}} L^{2}
\end{aligned}
$$

for the WC with the hexagonal lattice and in the longwavelength limit $k_{1}, k_{2} \ll 1 / a$. Here $L^{2}$ is the area of the system. Another important property of $S_{\alpha \beta}$ is a nonzero mean,

$$
\left\langle S_{\alpha \beta}(\mathbf{r})\right\rangle=\delta_{\alpha \beta} S_{0}
$$

Parameter $S_{0}$ is positive, in accordance with the pinning phenomenon: the crystal in its ground state is distorted in such a way that the electrons are preferentially situated near the minima of the random potential $U(\mathbf{r})$ where its curvature is positive. $S_{0}$ can be estimated to be

$$
S_{0} \simeq-\frac{V^{\prime \prime}(0)}{2 \pi \mu} \ln \left(\frac{R_{c}}{a}\right)
$$

using the same procedure as for deriving Eq. (10). Assuming that $\left|\mathbf{u}^{(0)}(\mathbf{r})-\mathbf{u}^{(0)}(0)\right|$ is "small," we expand the exponential in Eq. (19) to obtain

$$
\tilde{S}_{\alpha \beta}(\mathbf{q}) \simeq-\frac{i n_{e}}{L^{2}} \sum_{\mathbf{K}} K_{\alpha} K_{\beta} K_{\gamma} \int_{\mathbf{q}_{1}} u_{\gamma}^{(0)}\left(\mathbf{q}_{1}\right) \tilde{U}\left(\mathbf{q}-\mathbf{q}_{1}-\mathbf{K}\right)
$$

Combining Eqs. (10) and (23), averaging over the disorder, and using the inequality $H_{L}^{0}(q) \gg H_{T}^{0}(q)$, we get

$$
S_{0} \simeq-V^{\prime \prime}(0) \int_{\mathbf{q}}\left[H_{T}^{0}(q)\right]^{-1}
$$


which leads to Eq. 22). Here we again had to cut off the infrared logarithmic divergence by hand, at $q=q_{c}=$ $\pi / R_{c}$. More sophisticated approximation schemes such as the GVRM would implement this cutoff more gracefully but as long as we are not interested in the numerical factor in the argument of the log, the result is the same.

At this point we conclude the discussion of the ground state properties of the pinned WC, as we are now ready to address its dynamical response.

\section{FINITE-FREQUENCY RESPONSE: PERTURBATION THEORY AND QUALITATIVE CONSIDERATIONS}

To study the dynamics of the $\mathrm{WC}$ we introduce a time-dependent displacement field $\mathbf{u}$, which is the deviation of the total displacement from its ground-state value $\mathbf{u}^{(0)}(\mathbf{r})$. We will restrict ourselves to the harmonic approximation where the action is quadratic in $\mathbf{u}$. The response of a harmonic oscillator is the same in quantum and classical mechanics, so we can use the convenient imaginary-time formalism without compromising our original intention to treat the system classically. The resultant action contains two terms: one which describes a uniformly pinned WC,

$$
A_{0}=\frac{1}{2} \frac{1}{\hbar \beta} \sum_{\omega_{n}} \int_{\mathbf{q}} \mathbf{u}^{\dagger}\left(\mathbf{q}, i \omega_{n}\right)\left[\mathbf{D}^{0}\right]^{-1} \mathbf{u}\left(\mathbf{q}, i \omega_{n}\right),
$$

and the other which takes into account fluctuations in local pinning strength,

$$
A_{1}=\frac{1}{2} \frac{1}{\hbar \beta} \sum_{\omega_{n}} \int_{\mathbf{q}_{1}} \int_{\mathbf{q}_{2}} \mathbf{u}^{\dagger}\left(\mathbf{q}_{1}, i \omega_{n}\right) \delta \tilde{\mathbf{S}}\left(\mathbf{q}_{1}-\mathbf{q}_{2}\right) \mathbf{u}\left(\mathbf{q}_{2}, i \omega_{n}\right)
$$

Here $\beta=1 /\left(k_{B} T\right)$ is the inverse temperature, $\omega_{n}=$ $2 \pi n /(\hbar \beta)$ are the bosonic Matsubara frequencies, $\delta \tilde{\mathbf{S}} \equiv$ $\tilde{\mathbf{S}}-S_{0} \mathbf{I}, \mathbf{I}$ is the identity matrix, $\mathbf{D}^{0}=\mathbf{D}^{0}\left(\mathbf{q}, i \omega_{n}\right)$ is the phonon propagator of a uniformly pinned $\mathrm{WC}$ (cf. Refs. 10 and 22)

$$
\left[\mathbf{D}^{0}\right]^{-1}=\mathbf{R}_{\mathbf{q}}^{\dagger}\left[\begin{array}{cc}
H_{T}^{0}+S_{0}+\rho \omega_{n}^{2} & -\rho \omega_{n} \omega_{c} \\
\rho \omega_{n} \omega_{c} & H_{L}^{0}+S_{0}+\rho \omega_{n}^{2}
\end{array}\right] \mathbf{R}_{\mathbf{q}}
$$

$\rho=m_{e} n_{e}$ is the average mass density, and $\mathbf{R}_{\mathbf{q}}$ is the $O(2)$ rotation by angle $\arg \left(q_{x}+i q_{y}\right)$. We also define the disorder-averaged propagator $\mathbf{D}\left(\mathbf{q}, i \omega_{n}\right)$,

$$
\mathbf{D}\left(\mathbf{q}, i \omega_{n}\right)=\left\langle\frac{\int \mathcal{D} \mathbf{u} \mathcal{D} \mathbf{u}^{*} \mathbf{u}\left(\mathbf{q}, i \omega_{n}\right) \mathbf{u}^{*}\left(\mathbf{q}, i \omega_{n}\right) e^{-A}}{\left(\hbar \beta L^{2}\right) \int \mathcal{D} \mathbf{u} \mathcal{D} \mathbf{u}^{*} e^{-A}}\right\rangle,
$$

where $A \equiv A_{0}+A_{1}$ and $L^{2}$ is again the area of the system.
The quantity we set out to calculate is the af conductivity $\boldsymbol{\sigma}(q, \omega)$, which in this model is given by 10

$$
\boldsymbol{\sigma}(q, \omega)=-\left.i e^{2} n_{e}^{2} \omega \mathbf{D}\left(q, i \omega_{n}\right)\right|_{i \omega_{n} \rightarrow \omega+i 0} .
$$

The conductivity can also be expressed in terms of the phonon self-energy

$$
\mathbf{\Pi}(\mathbf{q}, \omega) \equiv S_{0} \mathbf{I}+\left.\left(\mathbf{D}^{-1}-\left[\mathbf{D}^{0}\right]^{-1}\right)\right|_{i \omega_{n} \rightarrow \omega+i 0}
$$

We are interested primarily in the case $q=0$ where the most general form of $\boldsymbol{\Pi}(\mathbf{q}, \omega)$ consistent with rotational symmetry is

$$
\Pi_{\alpha \beta}(0, \omega)=\delta_{\alpha \beta} \Pi(\omega)-i \varepsilon_{\alpha \beta} \rho \omega \omega_{c} f_{x y}(\omega),
$$

$f_{x y}(\omega)$ being the relative correction $\Delta \rho_{x y} / \rho_{x y}^{0}$ to the bare Hall resistivity $\rho_{x y}^{0}(\omega)=B /\left(n_{e} e c\right)$. Approximate calculations presented in this paper give $f_{x y}=0$, which leads us to believe that $f_{x y}(\omega)$ must be small for weak pinning. We choose to neglect such fine details and to assume that $f_{x y}$ vanishes 28 In this case $\Pi(0, \omega)$ is a scalar and $\sigma_{x x}(\omega)$ is given by

$$
\operatorname{Re} \sigma_{x x}(\omega)=e^{2} n_{e}^{2} \omega \operatorname{Im} \frac{\Pi(0, \epsilon)-\epsilon}{[\Pi(0, \epsilon)-\epsilon]^{2}-\epsilon \epsilon_{c}},
$$

where we introduced convenient "energy" variables

$$
\epsilon \equiv \rho \omega^{2}, \quad \epsilon_{c} \equiv \rho \omega_{c}^{2} .
$$

From Eq. (32) one can see that in strong magnetic fields, $\omega_{c} \gg \omega_{p}$, the power absorption in the uniform electric field takes place mainly within the frequency interval of width

$$
\Delta \omega_{p}=-\frac{\operatorname{Im} \Pi\left(0, \epsilon_{p}\right)}{\rho \omega_{c}}
$$

centered at $\omega=\omega_{p}$, where

$$
\omega_{p}=\sqrt{\frac{\epsilon_{p}}{\rho}}=\frac{\operatorname{Re} \Pi\left(0, \epsilon_{p}\right)}{\rho \omega_{c}} .
$$

The last equation is the implicit definition of $\epsilon_{p}$. In the following we will work predominantly with the "energy" variable $\epsilon$ rather than the frequency $\omega$.

At small $\epsilon$ the calculation of $\boldsymbol{\Pi}$ is a difficult problem but at large energies, $\epsilon \gg \epsilon_{p}$, the first Born approximation suffices. The only parameters needed to implement it are the mean and the variance of the matrix elements of $\mathbf{S}$ given by Eqs. (20) and (21). For $q \ll 1 / a$ we obtain that $\Pi_{\alpha \beta}(\mathbf{q}, \epsilon)=\delta_{\alpha \beta} \Pi(\epsilon)$, where

$$
\Pi(\epsilon)=S_{0}+V^{\prime \prime}(0) \int_{\mathbf{k}} \operatorname{tr} \mathbf{D}^{0}(\mathbf{k}, \epsilon) .
$$

$\Pi$ has both imaginary and real parts, corresponding to the broadening and the frequency shift of the pinning mode, Eqs. (34) and (35), respectively. The imaginary 
part comes solely from the pole $(\mathrm{s})$ of $\mathbf{D}^{0}(\mathbf{k}, \epsilon)$, i.e., the solutions of $\left[H_{T}^{0}(\mathbf{k})+S_{0}-\epsilon\right]\left[H_{L}^{0}(\mathbf{k})+S_{0}-\epsilon\right]-\epsilon \epsilon_{c}=0$. A more accurate expression for $\Pi$ can be obtained using the self-consistent Born approximation (SCBA),

$$
\Pi(\epsilon)=S_{0}+V^{\prime \prime}(0) \int_{\mathbf{k}} \operatorname{tr} \mathbf{D}(\mathbf{k}, \epsilon),
$$

as long as the full propagator $\mathbf{D}(\mathbf{k}, \epsilon)$ has a pole at $|k| \gg q_{c}=\pi / R_{c}$. Under this condition the diagrams with intersecting lines not included into the SCBA are suppressed, much like they are suppressed in a dirty metal with $k_{F} \gg l^{-1}, k_{F}$ being the Fermi momentum and $l$ being the mean free path. The indicated condition is satisfied when

$$
\epsilon \gg \min \left\{\Pi_{0}, \frac{\lambda}{\mu} \frac{\Pi_{0}^{2}}{\epsilon_{c}}\right\} .
$$

Here and below $\lambda$ is meant to be evaluated at $q=q_{c}$ unless it is indicated otherwise. Parameter $\Pi_{0}$, which can be estimated to be

$$
\Pi_{0} \sim \mu q_{c}^{2}
$$

represents the real part of the self-energy at the lowest $\epsilon$ allowed by inequality (38). $\Pi_{0}$ is smaller than $S_{0}$ by the logarithmic factor due to the partial cancellation between the first and the second terms in Eq. (37). This unfortunate cancellation leaves us only with the order of magnitude estimate (39).
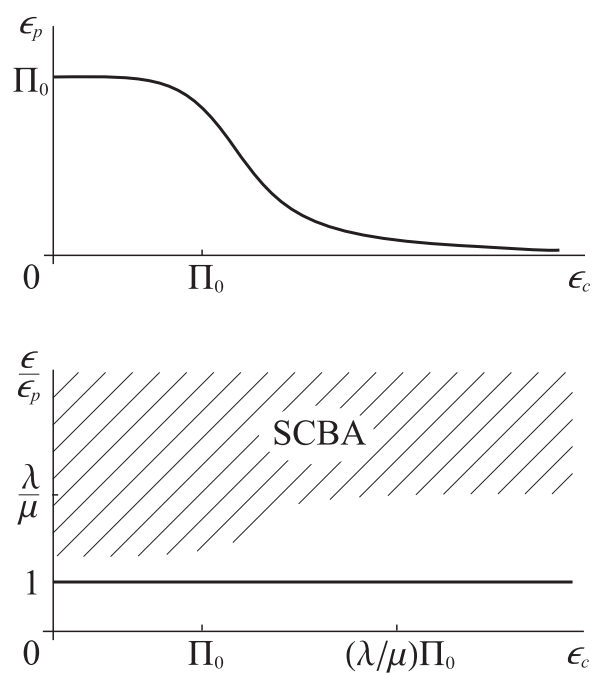

FIG. 3. Top: Pinning mode location as a function of the magnetic field. Bottom: SCBA domain of applicability.

When inequality (38) is violated, the SCBA becomes an uncontrolled approximation. The only SCBA result, which can presumably be trusted at such $\epsilon$, is a slow dependence of $\operatorname{Re} \Pi(\epsilon)$ on energy. This is because the real part of the integral in Eq. (37) is dominated by large $k$ 's where the lowest-order perturbation theory is valid $\mathrm{T}_{0}$ estimate $\epsilon_{p}$ we can take $\operatorname{Re} \Pi(\epsilon) \approx \Pi_{0}$, which yields 10

$$
\epsilon_{p} \sim \min \left\{\Pi_{0}, \frac{\Pi_{0}^{2}}{\epsilon_{c}}\right\}
$$

represented graphically in Fig. 3 together with the SCBA domain.

As one can see from this graph, for $\epsilon_{c}<\Pi_{0}$ (weak magnetic fields) there is no parametric separation between the SCBA domain and the curve $\epsilon=\epsilon_{p}$; therefore, the SCBA is qualitatively correct even along this curve where it predicts $\operatorname{Im} \Pi\left(\epsilon_{p}\right) \sim-\Pi_{0}$. Hence, $\Delta \omega_{p} \sim \omega_{p}$. The resultant broad absorption maximum is sketched in Fig. 4.

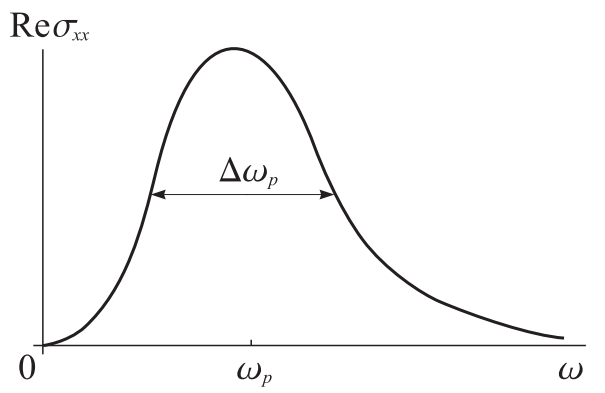

FIG. 4. Frequency dependence of the conductivity in weak magnetic fields.

If $\lambda$ and $\mu$ are comparable, the same argument works also in strong magnetic fields $\left(\epsilon_{c} \gg \Pi_{0}\right)$ where $\sigma_{x x}(\omega)$ looks qualitatively similar, except the position of the maximum depends on the magnetic field: $\omega_{p}=\omega_{p 0}^{2} / \omega_{c}$, where $\omega_{p 0}$ is the pinning frequency at $B=0$. On the other hand, if $\lambda \gg \mu$, as for the WC, the pinning mode is situated in the interior of a parametrically wide range of $\epsilon$ where the SCBA fails. Thus, the calculation of $\Delta \omega_{p}$ requires other methods. Later we will show that $\Delta \omega_{p}$ in strong magnetic fields is related to the low-frequency tail of $\sigma_{x x}(\omega)$ in the absence of the magnetic field. Although calculating the functional form of such a tail is another nontrivial problem (after all, it is beyond the SCBA!), this intriguing relation is sufficient to establish that the absorption line narrows down, in agreement with the experiments.14 17

We unfold our argument gradually over the remaining sections. In this section we start implementing this task by clarifying why $\sigma_{x x}(\omega)$ is virtually independent of the longitudinal stiffness $\lambda$ provided $\lambda \gg \mu$ and $B=0$.

Since action $A$ is quadratic in $\mathbf{u}_{L}$, the L-phonon degrees of freedom can be easily integrated out, leading to the effective Hamiltonian for the T-phonons,

$$
\mathbf{H}_{T}=\mathbf{H}_{T}^{0}+\mathbf{S}_{T}-\mathbf{S}_{X+}^{\dagger}\left(\mathbf{H}_{L}^{0}+\mathbf{S}_{L}-\epsilon \mathbf{I}\right)^{-1} \mathbf{S}_{X+},
$$

where $\mathbf{H}_{T}^{0}, \mathbf{H}_{L}^{0}$, and $\mathbf{S}_{i}$ should be understood as operators. The first two are diagonal in the basis of plane 
waves, with matrix elements $H_{T}^{0}(q)$ and $H_{L}^{0}(q)$, respectively; $\mathbf{S}_{i}$ 's have both diagonal and off-diagonal matrix elements:

$$
\begin{aligned}
& S_{L \mathbf{q q}^{\prime}}=\tilde{S}_{\alpha \beta}\left(\mathbf{q}-\mathbf{q}^{\prime}\right) \hat{q}_{\alpha} \hat{q}_{\beta}^{\prime}, \\
& S_{T \mathbf{q q}^{\prime}}=\left(\delta_{\alpha \beta} \operatorname{tr} \tilde{\mathbf{S}}-\tilde{S}_{\alpha \beta}\right) \hat{q}_{\alpha} \hat{q}_{\beta}^{\prime}, \\
& S_{X \mathbf{q q}^{\prime}}=\tilde{S}_{\alpha \beta}\left(\mathbf{q}-\mathbf{q}^{\prime}\right) \epsilon_{\beta \gamma} \hat{q}_{\alpha} \hat{q}_{\gamma}^{\prime} .
\end{aligned}
$$

Finally, $\mathbf{S}_{X+} \equiv \mathbf{S}_{X}+\sqrt{\epsilon \epsilon_{c}} \mathbf{I}$. Integrating out $\mathbf{u}_{L}$ is an exact algebraic transformation, which preserves the spectrum of the collective modes: the resolvent $\mathbf{D}_{T}(\epsilon)$ of the operator $\mathbf{H}_{T}-\epsilon \mathbf{I}$ has poles at the same $\epsilon$ as the full propagator. In fact, $\mathbf{D}_{T}$, which can be written in the form

$$
\mathbf{D}_{T}=\left(\left[\mathbf{D}_{T}^{*}\right]^{-1}-\mathbf{S}^{*}\right)^{-1},
$$

where

$$
\begin{aligned}
& \mathbf{D}_{T}^{*}=\left(\mathbf{H}_{T}^{0}+\mathbf{S}_{T}-\epsilon \mathbf{I}\right)^{-1} \\
& \mathbf{S}^{*}=\mathbf{S}_{X+}^{\dagger}\left(\mathbf{H}_{L}^{0}+\mathbf{S}_{L}-\epsilon \mathbf{I}\right)^{-1} \mathbf{S}_{X+},
\end{aligned}
$$

is nothing else than the T-T component of the full phonon propagator before the disorder averaging. The self-energy of the averaged propagator at $q=0$ is a scalar (see above); therefore, the T-T component determines the entire propagator for this particular $q$.

Let us now show that in zero magnetic field the mixing between the T- and L-phonons represented by $\mathbf{S}^{*}$ has little effect on $\mathbf{D}_{T}$. Indeed, in the diagrammatic expansion of $\left\langle\mathbf{D}_{T}(\epsilon)\right\rangle$ in powers of $\delta \mathbf{S}_{T}$ and $\mathbf{S}^{*}$, the typical momentum transfer $k$ for $\epsilon \lesssim \Pi_{0}$ is of the order of $q_{c}$. A single occurence of $\mathbf{S}^{*}$ contributes $\sim k^{2}\left|V^{\prime \prime}(0)\right| / \lambda k^{2} \sim(\mu / \lambda) \Pi_{0}$ to the self-energy, compared to $\sim \Pi_{0}$ from $\delta \mathbf{S}_{T}$. Diagrams with multiple occurences of $\mathbf{S}^{*}$ are suppressed by even higher powers of the small parameter $\mu / \lambda$. Thus, in the parameter range relevant for the observation of the pinning mode

$$
\left\langle\mathbf{D}_{T}\right\rangle(\mathbf{q}, \epsilon) \simeq\left\langle\mathbf{D}_{T}^{*}\right\rangle(\mathbf{q}, \epsilon) \equiv \frac{1}{H_{T}^{0}(q)+\Pi^{*}(\mathbf{q}, \epsilon)-\epsilon} .
$$

Note that $\mathbf{D}_{T}^{*}$ is determined only by the shear modulus $\mu$ and the disorder in the T-T channel $\delta \mathbf{S}_{T}$, while bulk modulus $\lambda$ drops out. It follows from this discussion that for $\lambda \gg \mu$ we can calculate the $q=0$ response pretending that the L-degree of freedom does not exist.

Let us indeed imagine that the L-phonons are forbidden and try to investigate the nature of the T-phonon eigenstates that would compose the pinning mode, i.e., the states that would respond to the uniform electric field (still at $B=0$ ). To do so we need to analyze the solutions of the eigenvalue problem for the single-particle Hamiltonian $\mathbf{H}^{*}=\mathbf{H}_{T}^{0}+\mathbf{S}_{T}$. In the uniformly pinned $\mathrm{WC}$, $\mathbf{S}_{T}=S_{0} \mathbf{I}$ and the eigenstates are just the plane waves labelled by momenta $\mathbf{k}$ of the Brillouin zone. In the actual random system the eigenstates are wavepackets of the plane waves with characteristic spread of momenta of the order of $L_{T}^{-1}$, where $L_{T} \sim v_{T} /|\operatorname{Im} \Pi|$ is the T-phonon mean free path, $v_{T}=\sqrt{\epsilon \mu}$ playing the role T-phonon "velocity." Since $\left|\operatorname{Im} \Pi\left(\epsilon_{p}\right)\right| \sim \epsilon_{p} \sim \mu q_{c}^{2}$, at $\epsilon \sim \epsilon_{p}$ we have $L_{T} \sim R_{c}$. Hence, the eigenstates that respond appreciably to a $q=0$ external drive are wavepackets built from $0<k<q_{c}$ plane waves. For such wavepackets the average $k$ is of the order of the inverse mean free path and the Ioffe-Regel criterion29 maintains that these states are localized. In other words, $R_{c}$ is not only the mean free path but also the localization length of the states within the pinning mode.

Let us define the $B=0$ T-phonon density of states,

$$
\nu(\epsilon)=\frac{1}{L^{2}}\left\langle\sum_{i} \delta\left(\epsilon-\epsilon_{i}\right)\right\rangle=\frac{1}{\pi} \int_{\mathbf{k}}\left\langle\mathbf{D}_{T}^{*}\right\rangle(\mathbf{k}, \epsilon) .
$$

At $\epsilon \gg \epsilon_{p}$ it tends to the bare value $\nu_{0}=(4 \pi \mu)^{-1}$, and at $\epsilon \sim \epsilon_{p}$ it is only slightly smaller. It is easy to see then that within an area $R_{c} \times R_{c}$ and energy interval $0<\epsilon<\epsilon_{p}$, there is typically only one (localized) state. The picture that emerges is illustrated in Fig. 5: we have a collection of localized states of roughly the same size (i.e., the inverse participation ratio) and roughly the same distance from each other in real space, both of the order of $R_{c}$. The broad distribution of shapes and sizes characteristic of localized states in disordered systems yields a large inhomogeneous broadening $\Delta \omega_{p} \sim \omega_{p}$ of the absorption line, as we found earlier based on a different line of reasoning.

In early works on pinning a heuristic imagery of "domains" was sometimes invoked 12 to describe the ground state structure. However, it was never clear where to draw the boundaries between different domains. This difficulty is partially resolved in our picture of phonon localization where domains can be defined as areas where individual localized phonon wavefunctions are appreaciable. However, modern understanding of the subject reviewed in the previous section no longer appeals to any "domains," and so this interpretation may be regarded as a historic sentiment.

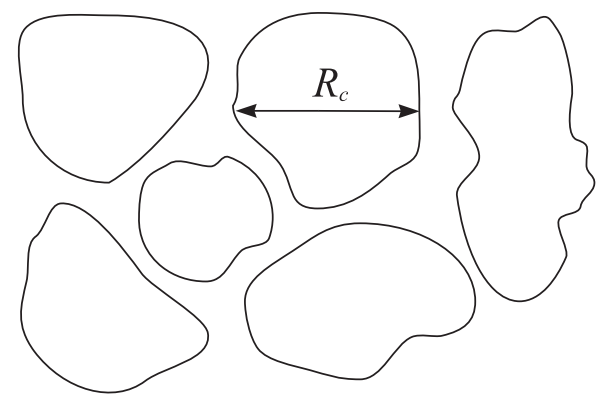

FIG. 5. Sketch of spacial distribution of T-phonon states contributing to the pinning mode at $B=0$.

One more historic comment is in order. Previous work 30 on phonon localization found that the phonon eigenstates remain extended even in the $\omega \rightarrow 0$ limit, seemingly in contradiction to our results. In fact, there 
is no contradiction because the model we study here and the conventional formulations, where disorder originates from the defects of the crystalline lattice, e.g., substitutions by impurity atoms, are quite different. While in those conventional models the defects move with the lattice and their influence rapidly diminishes at small k's, the pinning potential for the $\mathrm{WC}$ is static, so it is not affected by the lattice motion. It couples to the phonons via the matrix $\mathbf{S}$ whose fluctuations have approximately the same rms magnitude at all k's within the Brillouin zone, see Eq. (20). This is the reason why in our case the disorder effects are much stronger, causing the localization of the phonon eigenstates. Actually, our model has more in common with those of noninteracting electrons in dirty metals and semiconductors. (At the oneparticle level the distinction between the bosonic statistics of phonons and the fermionic statistics of electrons is irrelevant).

Concluding this section, we would like to reiterate one of its main results, that the presence (or absence) of the stiff L-phonon degree of freedom affects the $B=0, q=0$ response only weakly. Naturally, it makes the fate of the L-phonons seem quite mysterious. The finite- $B$ dynamical response has also been barely touched upon. These gaps in understanding will be filled in the next section.

\section{SCATTERING OF L-PHONONS AND PINNING MODE LINEWIDTH IN A FINITE MAGNETIC FIELD}

In the previous section we discussed the scattering and localization of the T-component of WC lattice vibrations. Let us now turn to the L-component. This time we integrate out $\mathbf{u}_{T}$ to obtain the effective Hamiltonian for the L-phonons, the corresponding Green's function, and the self-energy:

$$
\begin{aligned}
& \mathbf{H}_{L}=\mathbf{H}_{L}^{0}+\mathbf{S}_{L}-\mathbf{S}_{X+}\left(\mathbf{H}_{T}^{0}+\mathbf{S}_{T}-\epsilon \mathbf{I}\right)^{-1} \mathbf{S}_{X+}^{\dagger}, \\
& \mathbf{D}_{L}=\left(\mathbf{H}_{L}-\epsilon \mathbf{I}\right)^{-1}, \\
& \boldsymbol{\Pi}_{L}(\epsilon)=\left\langle\mathbf{D}_{L}\right\rangle^{-1}-\left(\mathbf{H}_{L}^{0}-\epsilon \mathbf{I}\right) .
\end{aligned}
$$

Since the self-energy $\boldsymbol{\Pi}(\mathbf{q}, \epsilon)$ of the original system (before integrating out $\mathbf{u}_{T}$ ) is a scalar at $\mathbf{q}=0, \Pi_{L}$ must obey the relation

$$
\Pi_{L}(0, \epsilon)=\Pi(0, \epsilon)-\frac{\epsilon \epsilon_{c}}{\Pi(0, \epsilon)-\epsilon}
$$

Our first task is to reproduce the results of Sec. III by demonstrating that in sufficiently weak magnetic fields $\Pi_{L}$ indeed has the above form with $\Pi \simeq \Pi^{*}$.

There are two types of processes that contribute to $\Pi_{L}$ : the intraband scattering (L-L channel) and the scattering through an intermediate T-state (L-T-L channel). They originate from the second and the third terms of $\mathbf{H}_{L}$, respectively. The intraband scattering will be treated in more detail in Appendix A where using a combination of the renormalization group and instanton methods we show that it is exponentially small. For our current purposes a weaker result is sufficient, that the intraband contribution $\Pi_{L \rightarrow L}$ to $\Pi_{L}$ is much smaller than $\Pi_{0}$. This can be established without detailed calculations. Indeed, the effective random potential $\mathbf{S}_{L}$ in the L-L channel has basically the same mean and rms fluctiations as $\mathbf{S}_{T}$; therefore, $\mathbf{S}_{L}$ and $\mathbf{S}_{T}$ can be regarded as disorder of approximately the same strength. Then the alluded inequality $\left|\Pi_{L \rightarrow L}\right| \ll|\Pi| \sim \Pi_{0}$ follows simply from the fact that L-phonons have much steeper bare dispersion relation $\epsilon(q)=\lambda(q) q^{2}$ than T-phonons and are scattered much weaker by disorder of the same strength. It is legitimate to neglect the L-L scattering altogether by replacing $\mathbf{S}_{L}$ with its average value $S_{0} \mathbf{I}$, and to concentrate exclusively on the L-T-L processes. In this case, much like for a dirty semiconductors with two bands of carriers, 31 the diagrammatic expansion of $\Pi_{L}$ can be formulated as the sum of all one-particle irreducible (1PI) graphs involving $\mathbf{S}_{X+}$ and $\mathbf{D}_{T}$, see Eqs. (43 45) and Fig. 6a-b.

(a)

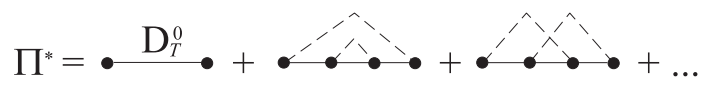

(b)

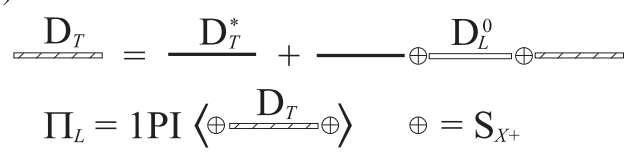

(c)

(d)

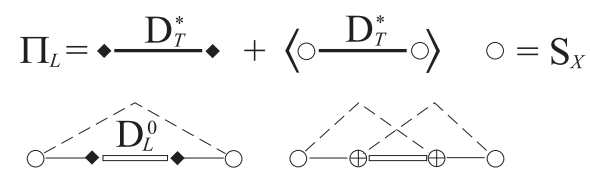

FIG. 6. (a) Diagrams for $\Pi^{*}$. Filled circles and solid lines represent $\delta \mathbf{S}_{T}$ and $\mathbf{D}_{T}^{0} \equiv\left(\mathbf{H}_{T}^{0}+S_{0} \mathbf{I}-\epsilon \mathbf{I}\right)^{-1}$, respectively. Dashed lines symbolize contractions. (b) Diagrammatic definitions of $\mathbf{D}_{T}$ and $\Pi_{L}$. (c) Diagrams without $\mathbf{S}^{*}$. Diamonds represent factors $i \sqrt{\epsilon \epsilon_{c}}$. (d) Some representive diagrams for $\Pi_{L}$ not included in (c).

The crucial point substantiated below is that $\mathbf{S}^{*}$ can be neglected, i.e., that $\mathbf{D}_{T}$ can be replaced by $\mathbf{D}_{T}^{*}$, provided $\lambda \gg \mu$ and the magnetic field is not too strong,

$$
\epsilon_{c} \ll(\lambda / \mu) \Pi_{0} .
$$

For such $\epsilon_{c}$ only two graphs contribute to $\Pi_{L}$ (Fig. 6. which is a significant simplification. However, we still have to specify how to average over the disorder. Naive averaging of $\mathbf{S}_{X}$ and $\mathbf{D}_{T}^{*}$ separately from each other yields

$$
\Pi_{L}(\mathbf{q}, \epsilon)=S_{0}-\epsilon \epsilon_{c}\left\langle\mathbf{D}_{T}^{*}\right\rangle(\mathbf{q}, \epsilon)+V^{\prime \prime}(0) \int_{\mathbf{k}}\left\langle\mathbf{D}_{T}^{*}\right\rangle(\mathbf{k}, \epsilon)
$$

but it is certainly not accurate because $\mathbf{S}_{X}$ and $\delta \mathbf{S}_{T}$ are not independent. Indeed, their two-point correlator 


$$
\begin{aligned}
\left\langle S_{X \mathbf{q}_{1} \mathbf{q}} S_{T \mathbf{q}_{2} \mathbf{q}_{3}}\right\rangle & \propto\left(\hat{\mathbf{q}}_{1} \hat{\mathbf{q}}_{2}\right)\left(\hat{\mathbf{q}}_{3} \hat{\mathbf{q}}\right)+\left(\hat{\mathbf{q}}_{1} \hat{\mathbf{q}}_{3}\right)\left(\hat{\mathbf{q}}_{2} \hat{\mathbf{q}}\right) \\
& +\left(\hat{\mathbf{q}}_{1} \hat{\mathbf{q}}\right)\left(\hat{\mathbf{q}}_{3} \hat{\mathbf{q}}_{2}\right)
\end{aligned}
$$

is in general nonvanishing.

In the special case of $\mathbf{q}=0$ a further progress is possible. Comparing the above equation with

$$
\begin{aligned}
\left\langle S_{T \mathbf{q}_{1} \mathbf{q}} S_{T \mathbf{q}_{2} \mathbf{q}_{3}}\right\rangle & \propto\left(\hat{\mathbf{q}}_{1} \hat{\mathbf{q}}_{2}\right)\left[\hat{\mathbf{q}} \times \hat{\mathbf{q}}_{3}\right] \hat{\mathbf{z}}+\left(\hat{\mathbf{q}}_{1} \hat{\mathbf{q}}_{3}\right)\left[\hat{\mathbf{q}} \times \hat{\mathbf{q}}_{2}\right] \hat{\mathbf{z}} \\
& +\left(\hat{\mathbf{q}}_{1} \hat{\mathbf{q}}\right)\left[\hat{\mathbf{q}}_{3} \times \hat{\mathbf{q}}_{2}\right] \hat{\mathbf{z}},
\end{aligned}
$$

we observe that the former is transformed into the latter by the replacement $\mathbf{q} \rightarrow[\hat{\mathbf{z}} \times \mathbf{q}]$. If the incoming momentum $\mathbf{q}$ is zero, rotating it by $\pi / 2$ has no effect. Thus, for $\mathbf{q}=0$ we can replace $\mathbf{S}_{X}$ in the second diagram of Fig. [ 6 c by $\delta \mathbf{S}_{T}$, after which it becomes identical to the totality of diagrams in Fig. 6a for $\Pi^{*}$; therefore,

$$
\Pi_{L}(0, \epsilon)=\Pi^{*}(0, \epsilon)-\frac{\epsilon \epsilon_{c}}{\Pi^{*}(0, \epsilon)-\epsilon} .
$$

As explained in Sec. III, $\Pi^{*} \simeq \Pi$; hence, we will succeed in reproducing Eq. (51) as soon as we show that inequality (52) is indeed the relevant condition for dropping $\mathbf{S}^{*}$ in the diagrammatic series for $\Pi_{L}$. To do so we develop one step further the argument of Sec. III. One-particle irreducible diagrams containing $\mathbf{S}^{*}$ have at least one Lphonon line with typical momentum transfer $k \sim q_{c}$, see Fig. 6d. If $\epsilon_{c}=0$ such diagrams are suppressed by at least a factor of $\mu / \lambda$, as we found previously in Sec. IIII. However, if $\epsilon_{c} \neq 0$, these diagrams also generate terms of the order of

$$
\sqrt{\epsilon \epsilon_{c}} \frac{1}{H_{L}^{0}(k)} \sqrt{\epsilon \epsilon_{c}} \sim \frac{\epsilon \epsilon_{c}}{\lambda q_{c}^{2}},
$$

which act as an additional self-energy correction to $\mathbf{D}_{T}^{*}$ in Fig. 6ic. They can be approximately accounted for if we replace $\epsilon$ in the argument of $\Pi^{*}$ in Eq. (56) by a renormalized value $\tilde{\epsilon}$,

$$
\begin{aligned}
\Pi_{L}(0, \epsilon) & =\Pi^{*}(0, \tilde{\epsilon})-\frac{\epsilon \epsilon_{c}}{\Pi^{*}(0, \tilde{\epsilon})-\epsilon}, \\
\tilde{\epsilon} & =\epsilon+C_{1} \frac{\epsilon \epsilon_{c}}{\lambda q_{c}^{2}}, \quad C_{1} \sim 1 .
\end{aligned}
$$

The difference between $\epsilon$ and $\tilde{\epsilon}$ can be safely ignored as long as the inequality (52) is satisfied, in which case Eq. $(56)$ is asymptotically exact. In stronger fields Eq. (58) applies, which gives only the order of magnitude of $\Pi_{L}$ because of the uncertainty in the parameter $C_{1}$.

The meaning of Eqs. (56) and (58) can be elucidated returning to the the original formulation where both $\mathrm{T}$ and L-degrees of freedom are present:

$$
\begin{aligned}
\Pi(0, \epsilon) & =\Pi^{*}(0, \epsilon), \quad \epsilon_{c} \ll(\lambda / \mu) \Pi_{0}, \\
& =\Pi^{*}(0, \tilde{\epsilon}), \quad \epsilon_{c} \gg(\lambda / \mu) \Pi_{0} .
\end{aligned}
$$

We see that $\Pi(0, \epsilon)$ remains magnetic field independent up to rather high $B$. As explained above, this occurs because the enhancement of the L-T mixing due to the
Lorentz force is strongly impeded by the disparity of the two elastic moduli.

Let us now evaluate the consequences of the obtained equations (60a) and (60b). In the intermediate field range, $\Pi_{0} \ll \epsilon_{c} \ll(\lambda / \mu) \Pi_{0}$, where Eq. (60a) applies, the consequences are two-fold: the suppression of $\epsilon_{p}$, which is well known, and the suppression of the pinning mode linewidth, which is nontrivial. Indeed, since $\epsilon_{p}$ becomes much less than $\Pi_{0}$ [Eq. (40)], it slips into the low-energy tail of the zero-field phonon spectrum where $\operatorname{Im} \Pi \ll \Pi_{0} ;$ hence, $\Delta \omega_{p} \ll \omega_{p}$. The connection between the linewidth of the absorption line in strong magnetic fields and the low-frequency phonon modes in zero magnetic field, which we just established, is the keystone of the present paper.

The properties of the zero-field soft modes will be discussed in more detail in Sec. $\mathrm{V}$ where we argue that they give rise to the power-law dependence of $\operatorname{Im} \Pi$ on $\epsilon$,

$$
\operatorname{Im} \Pi(\epsilon) \simeq-C_{2} \Pi_{0}\left(\epsilon / \Pi_{0}\right)^{s}, \quad \epsilon \ll \Pi_{0},
$$

with exponent $s=3 / 2$ and numerical prefactor $C_{2} \sim 1$. Combining Eqs. (34 35) and (60 61), we find for the intermediate- $B$ regime:

$$
\begin{aligned}
& \sigma_{x x}(\omega)=-i \frac{e^{2} n_{e} \omega}{m \omega_{p 0}^{2}} \frac{1-i f_{1}(\omega)}{\left[1-i f_{1}(\omega)\right]^{2}-\left(\omega \omega_{c} / \omega_{p 0}^{2}\right)^{2}}, \\
& f_{1}(\omega) \simeq\left\{\begin{array}{cc}
C_{2}\left(\omega / \omega_{p 0}\right)^{2 s}, & \omega \ll \omega_{p 0}, \\
\text { const }, & \omega_{p 0} \ll \omega \ll \omega_{c},
\end{array}\right. \\
& \omega_{p 0} \ll \omega_{c} \ll \omega_{p 0} \sqrt{\lambda / \mu} .
\end{aligned}
$$

In this regime the absorption line narrows down according to Eq. (2), which we reproduce here for convenience:

$$
\Delta \omega_{p} / \omega_{p} \sim\left(\omega_{p} / \omega_{c}\right)^{s} .
$$

The high-field regime is described by Eq. (60b). In this case the relative linewidth is field-independent and is of the order of

$$
\Delta \omega_{p} / \omega_{p} \sim(\mu / \lambda)^{s}
$$

as given by Eq. (3) and illustrated in Fig. 1. Although Eq. (60b) was derived with much less rigor than Eq. 600 , the saturation of $\Delta \omega_{p} / \omega_{p}$ in strong fields is certainly to be expected. Indeed, in strong fields the dynamics is dominated by the Lorentz force; therefore, $\epsilon$ must enter in the combination $\epsilon \epsilon_{c}$, not by itself. But then a phonon eigenstate, which for a given (large) $\epsilon_{c}=\epsilon_{c}^{*}=\rho\left(\omega_{c}^{*}\right)^{2}$ has an energy $\epsilon_{i}$, is also an eigenstate of the system for larger $\epsilon_{c}$, with the eigenvalue $\epsilon_{i}\left(\epsilon_{c}^{*} / \epsilon_{c}\right)$. Hence, as the magnetic field increases, all relevant eigenfrequencies scale inversely proportional to $\omega_{c}$, while the conductivity varies in the self-similar way,

$$
\sigma_{x x}(\omega)=\frac{\omega_{c}^{*}}{\omega_{c}} \sigma_{x x}^{*}\left(\frac{\omega \omega_{c}}{\omega_{c}^{*}}\right),
$$


and posseses constant $\Delta \omega_{p} / \omega_{p}$. The explicit expression for $\sigma_{x x}(\omega)$ is similar to Eq. (63),

$$
\begin{aligned}
\sigma_{x x}(\omega) & =-i \frac{e^{2} n_{e} \omega}{m \omega_{p 0}^{2}} \frac{1-i f_{2}(\omega)}{\left[1-i f_{2}(\omega)\right]^{2}-\left(\omega \omega_{c} / \omega_{p 0}^{2}\right)^{2}}, \\
f_{2}(\omega) & \simeq\left\{\begin{array}{cc}
(\omega / \Omega)^{2 s}, & \omega \ll \Omega, \\
\text { const }, & \Omega \ll \omega \ll \omega_{c},
\end{array}\right. \\
\Omega & =C_{3} \frac{\omega_{p 0}^{2}}{\omega_{c}} \sqrt{\lambda / \mu}, \quad \omega_{c} \gg \omega_{p 0} \sqrt{\lambda / \mu},
\end{aligned}
$$

where $C_{3} \sim 1$.

Formulas (63) and $(69)$ are our final results. They describe the entire lineshape of the pinning mode both in intermediate and strong magnetic fields. However, their derivation was presented in diagrammatic rather than physical terms. Next, we will give an alternative derivation, which elucidates the physics of the line narrowing and also helps to clarify the structure of the phonon eigenstates that compose the pinning mode.

We will start with the qualitative picture of localized T-phonons developed in Sec. III and add a new ingredient, the stiff L-degree of freedom. It is clear that an admixture of the L-component produced by a joint action of the disorder and the Lorentz force makes phonon eigenstates much more extended in real space. Unlike the softer T-phonons, the stiffer L-ones cannot be confined in small areas of size $R_{c}$. In order to understand the large scale structure of the eigenstates, we can coarse-grain the system by integrating out the degrees of freedom on the spacial scales between $a$ and $R_{c}$. An insight on the form of the effective Hamiltonian $\mathbf{H}_{L}^{\text {eff }}$ after the coarsegraining is given by the spectral decomposition of the matrix element

$$
\left\langle\mathbf{r}\left|\frac{1}{\mathbf{H}_{T}^{0}+\mathbf{S}_{T}-\epsilon \mathbf{I}}\right| \mathbf{r}^{\prime}\right\rangle=\sum_{i} \frac{u_{T i}(\mathbf{r}) u_{T i}^{*}\left(\mathbf{r}^{\prime}\right)}{\epsilon_{i}-\epsilon}
$$

Here $\epsilon_{i}$ and $u_{T i}(\mathbf{r})$ are the eigenvalues and the eigenfunctions of localized T-phonons. Since each of $u_{T i}$ is localized within an area of size $R_{c}$, we expect that after the coarse-graining the numerators $u_{T i}(\mathbf{r}) u_{T i}^{*}\left(\mathbf{r}^{\prime}\right)$ transform into local operators $R_{c}^{-2} \delta\left(\mathbf{r}-\mathbf{r}^{\prime}\right) \delta\left(\mathbf{r}-\mathbf{r}_{i}\right)$, where $\mathbf{r}_{i}$ is the center-of-gravity of the $i$ th mode. As for the denominators, the discussion in Sec. III shows that in weak and intermediate magnetic fields [defined by the inequality (52)], $\epsilon_{i}$ 's should remain unaffected. Finally, naive averaging of $\mathbf{S}_{L}$ in Eq. (7) yields $S_{0} \mathbf{I}$ but the interaction with high- $k$ T-modes, which can be calculated within the SCBA-type perturbation theory, renormalizes $S_{0}$ to $\Pi_{0}$. The resultant coarse-grained Hamiltonian is

$$
\mathbf{H}_{L}^{e f f}=\mathbf{H}_{L}^{0}+\Pi_{0} \mathbf{I}-\sum_{i} \frac{c_{i} \Pi_{0}^{2}+\epsilon \epsilon_{c}}{\epsilon_{i}-\epsilon} R_{c}^{2} \delta\left(\mathbf{r}-\mathbf{r}_{i}\right),
$$

where $c_{i} \sim 1$ are random and $\epsilon_{i} \in\left(0,2 \Pi_{0}\right)$. Without losing essential physics, we can assume that $\mathbf{r}_{i}$ 's form a regular square lattice with lattice constant $R_{c}$.
What are the properties of the obtained lattice model? One of them is the Lorentzian tails of the distribution function $P(U)$ of the on-site disorder terms $U_{i} \equiv$ $\left(c_{i} \Pi_{0}^{2}+\epsilon \epsilon_{c}\right) /\left(\epsilon-\epsilon_{i}\right)$. For example, if $\epsilon \lesssim \epsilon_{p}$, then

$$
P(U) \sim \frac{\Pi_{0}^{2} R_{c}^{2} \nu(\epsilon)}{U^{2}}, \quad|U| \rightarrow \infty,
$$

where $\nu(\epsilon)$ is the T-phonon density of states introduced in Sec. III. Clearly, the variance $\left\langle U^{2}\right\rangle$ of the on-site disorder is unbounded. One can therefore expect much stronger disorder effects than in the case of a Gaussian distribution with the same typical values of $U_{i}$, i.e., same $\langle\ln |U|\rangle$. Let $L_{L}$ be the mean-free path of L-component of the low-energy phonons. As explained in Sec. IIII, IoffeRegel criterion 29 suggests that $L_{L}$ is simultaneously their localization length. For weak Gaussian disorder and unscreened Coulomb interactions $L_{L}$ is exponentially large, see Appendix A. Now we will show that in the presence of the long tails (73), $L_{L}$ is only as a power-law function of the disorder strength. The crucial point is that the scattering is dominated by a few largest $U_{i}$ 's. As soon as we realize this, we can replace $P(U)$ by the Cauchy distribution

$$
P(U)=\frac{1}{\pi} \frac{\Gamma}{\left(U-U_{0}\right)^{2}+\Gamma^{2}}, \quad \Gamma(\epsilon) \sim \Pi_{0}^{2} R_{c}^{2} \nu(\epsilon)
$$

on the grounds that it has the same tails. At this point we make a reasonable assumption that correlations among $U_{i}$ 's at different sites can be neglected, and arrive at the famous Lloyd-model. The self-energy can now be calculated exactly, 32

$$
\Pi_{L}(\epsilon)=\Pi_{0}-U_{0}(\epsilon)-i \Gamma(\epsilon),
$$

while the mean-free path is the solution of the equation $H_{L}^{0}\left(L_{L}^{-1}\right)=\left|\operatorname{Im} \Pi_{L}\right|$, which gives $L_{L} \sim Y / \Gamma$.

To compare with our earlier results we need to know $\nu$ and $U_{0}$. The integral in Eq. (47) is determined by $|\mathbf{k}| \lesssim q_{c} ;$ therefore,

$$
\nu(\epsilon) \sim q_{c}^{2} \frac{\operatorname{Im} \Pi^{*}(\epsilon)}{\Pi_{0}^{2}} \sim \mu^{-1}\left(\frac{\epsilon}{\Pi_{0}}\right)^{s}, \quad \epsilon \lesssim \Pi_{0} .
$$

The estimate of $U_{0}$ is $U_{0} \sim \epsilon \epsilon_{c} / \Pi_{0}$. Combining these together, we find that Eq. (75) is in agreement with Eq. (56), which validates our mapping onto the Lloyd model.

To describe the dominant scattering mechanism in the Lloyd model notice that $\operatorname{Im} \Pi_{L}$ comes from the inhomogeneous broadening of the phonon energies, i.e., the energy shifts $\delta \epsilon$ caused by on-site disorder $U_{i}$. For each localized L-phonon the largest energy shift comes from a site with largest $U_{i}$ within the localization area $L_{L} \times L_{L}$, which is typically $U_{i} \sim\left(L_{L} / R_{c}\right)^{2} \Gamma$ for the Cauchy distribution (74). Since the amplitude of the L-phonon wavefunction at the position of the oscillator is $u_{L} \sim 1 / L_{L}$, the resultant energy shift is $\delta \epsilon \sim U_{i} u_{L}^{2} R_{c}^{2} \sim \Gamma$. The energy shifts from other sites are subdominant; thus, 
$\operatorname{Im} \Pi_{L} \sim \Gamma$, in agreement with the exact result, Eq. 75 . In the original formulation large $U_{i}$ come from the localized T-oscillators whose energy $\epsilon_{i}$ is almost in resonance with $\epsilon$, i.e., $\left|\epsilon_{i}-\epsilon\right| \sim 1 / \nu(\epsilon) L_{L}^{2}$. A few of such resonant scatterers will localize the L-component on the scale of the mean-free path $L_{L}$. The proposed picture resembles the situation in a crystal pinned by strong dilute impurities, the role of impurities played by the resonant sites.

The structure of a typical phonon is illustrated in Fig. 7. It involves of the order of $\left(L_{L} / R_{c}\right)^{2}$ T-modes of Fig. foscillating with the same frequency imposed by the mutual coupling mediated by the L-phonon. The oscillation amplitude is roughly the same for all the T-modes except for a few resonant ones, where it is much larger. These resonant sites "drain" the energy of the L-degree of freedom thereby localizing it.

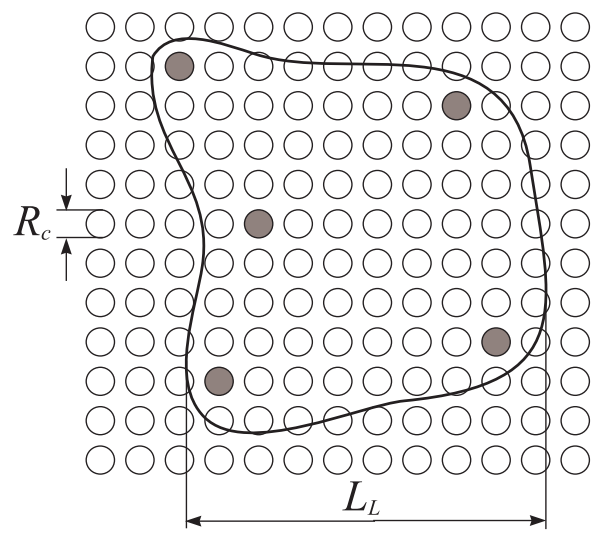

FIG. 7. Schematic structure of the localized phonon eigenstate in the crystal with both $\mathrm{T}$ - and L-degrees of freedom present. Circles represent the T-modes of Fig. the resonant modes are shaded.

Let us now describe the evolution of the phonon eigenstates as the magnetic field increases. Since the pinning mode frequency goes down, lower and lower frequency resonant sites are required to effectively scatter and localize the mixed L-T phonon vibrations. Such sites are therefore the soft modes, the oscillators of unusually low frequency, which shape up the tails of the zero-field phonon spectrum (Lifshitz tails). The soft modes appear due to very rare, peculiar disorder configurations. The lower the frequency, the mode dilute in real space they are. Correspondingly, the mean-free path (localization length) of the magnetophonons becomes larger and larger. The narrowing of the absorption line is then similar to the motional narrowing phenomenon. Eventually, in very strong magnetic fields, the enhancement of the L-T mixing by the Lorentz force start to diminish the frequencies of the localized T-phonons. At this point slipping of the pinning frequency $\omega_{p}$ deeper into the soft-mode tail stops. The wavefunctions of the phonons cease to change, only their eigenfrequencies continue decreasing in inverse proportion to the magnetic field. As a result, $\Delta \omega_{p} / \omega_{p}$ remains constant.
The physical structure of the pinned phonon modes being clarified, the only important questions that remain to be considered are the nature of the soft modes and the derivation of Eq. (61). This will be the subject of the next section.

\section{SOFT MODES}

It is clear that the soft phonon modes must come from rare places where pinning is unusually weak. The actual calculation of the density of states $\nu(\epsilon)$ in the low- $\epsilon$ tail is however nontrivial. The method most suitable for the task seems to be the method of optimal fluctuation. It has been successfully employed for calculation of the Lifshitz tails in other disordered systems, 20 where it proves to be asymptotically exact. The method is based on the following idea. The energy $\epsilon$ of a given eigenstate is determined in a complicated way by the distribution of the random potential within the entire area supporting the eigenstate and so there are many different random potential configurations, which give the same eigenenergy. If $\epsilon$ is small, any such configuration is untypical, i.e., a fluctuation of some sort. The method is based on the assumption that certain fluctuations have much higher probability than the rest and dominate the quantity of interest, $\nu(\epsilon)$ in this case. The objective is then to design such an optimal fluctuation and evaluate its probability.

Let us mention one type of fluctuation, which is not optimal. This is a configuration where the random potential is strongly suppressed in a large area $L \times L$, where $L \sim$ $\sqrt{\mu / \epsilon}$. (It may be useful to recall at this point that we are investigating the soft modes of a system where L-phonons are integrated out). The probability of such a fluctuation can be estimated by mentally dividing the area into uncorrelated blocks of size $R_{c} \times R_{c}$ and multiplying together the probabilities $\sim \epsilon / \Pi_{0}$ that the random potential is suppressed within each block. The total probability is exponentially small $\sim \exp \left[-\left(\Pi_{0} / \epsilon\right) \ln \left(\Pi_{0} / \epsilon\right)\right]$. This is a general situation for fluctuations spread over a large area in real space. Thus, the optimal fluctuation must be of the smallest possible size. It is easy to see that this size is $R_{c}$. Indeed, to get a small phonon eigenenergy $\epsilon$ in a small volume, the positive "kinetic energy" $\mu L^{-2}$ must be accurately compensated by the negative "potential energy" $\left\langle\delta \mathbf{S}_{T}\right\rangle_{L}$. The fluctuations of the latter averaged over the area $L \times L$ are of the order of $\Pi_{0}\left(R_{c} / L\right)$. Thus, for $L \sim R_{c}$ the cancellation of $\mu L^{-2}$ can be done by a typical fluctuation of $\delta \mathbf{S}_{T}$ and the probability of such an event would not have any exponential suppression. Instead, we expect the power-law dependence of $\nu(\epsilon)$ on $\epsilon$, Eq. (76).

In order to possess a soft mode, the system must have a soft direction, i.e., a certain collective coordinate $X$ such that the total energy of the system $E$ as a function of $X$ has a very shallow minimum. Let $X=0$ be the ground state. In its vicinity $E$ should be Taylor-expandable, 


$$
E(X)=\frac{\alpha}{2} X^{2}+\beta X^{3}+\gamma X^{4}+\ldots
$$

The coefficient $\alpha$ has the meaning of an effective spring constant of the local oscillator, while the phonon energy $\epsilon$ is determined by the ratio of the spring constant and the effective mass. The latter is proportional to the area involved in the oscillations. As we argued above, this area is of the order of $R_{c}^{2}$ for all $\epsilon<\epsilon_{p}$. Therefore, $\alpha$ should scale linearly with $\epsilon$ in the limit $\epsilon \rightarrow 0$,

$$
\alpha=C_{4} \epsilon
$$

If we find a way to estimate the probability of such untypically small $\alpha$, we will succeed in calculating the exponent $s$ of the power-law function $\nu(\epsilon)$.

This kind of calculation is hardly possible without specifying $X$. Actually, ascribing the precise meaning to $X$ amounts more or less to designing the optimal fluctuation. At the moment we do not have a good understanding how to do it in the $2 \mathrm{D}$ case. However, in one dimension the general structure of the calculation is clear. We are guided by the earlier work on the subject by Feigelman et al.33 and by Aleiner and Ruzin 34

In the case of a weakly pinned $1 \mathrm{D}$ elastic chain, the role of the desired collective coordinate $X$ can be played by the elastic displacement field $u$ at an arbitrary point in the middle of the chain. This point divides the system into two parts, which communicate only through the single variable $u$. For any fixed $u$ serving as a boundary condition, we can find separately the ground state energies $E_{<}(u)$ and $E_{>}(u)$ of the left and the right halves of the chain. The ground state of the whole system is determined by minimizing the sum $E(u)=E_{<}(u)+E_{>}(u)$ with respect to $u$. Both $E_{<}(u)$ and $E_{>}(u)$ are periodic function of $u$ with the period equal to the lattice constant $a$. On their period they have several (typically, of the order of $a / \xi$ ) minima and maxima. The beautiful idea of Aleiner and Ruzin 34 (totally overlooked in Ref. 33) was that the most efficient way to generate a soft mode is via a frustration, when a maximum of $E_{<}(u)$ occurs near a minimun of $E_{>}(u)$. In this case the second derivative of $E$, i.e., $\alpha=E^{\prime \prime}$, can be very small even though each of the second derivatives of $E_{<}(u)$ and $E_{>}(u)$ are typical. The next step is to show that the probability density distribution $P(\alpha)$ of $\alpha$ at local minima of $E$ vanishes linearly with $\alpha$ in the limit $\alpha \rightarrow 0$. Indeed,

$$
\begin{aligned}
P(\alpha) & =\left.\left\langle\delta\left(E^{\prime \prime}-\alpha\right)\right\rangle\right|_{E^{\prime}=0} \\
& =\left\langle\frac{1}{a} \int_{0}^{a} d u \delta\left(E^{\prime \prime}-\alpha\right) \delta\left(E^{\prime}\right)\left|\frac{\partial E^{\prime}}{\partial u}\right|\right\rangle \\
& \rightarrow|\alpha|\left\langle\delta\left(E^{\prime \prime}\right) \delta\left(E^{\prime}\right)\right\rangle \\
& =|\alpha|\left\langle\delta\left(E_{<}^{\prime \prime}+E_{>}^{\prime \prime}\right) \delta\left(E_{<}^{\prime}+E_{>}^{\prime}\right)\right\rangle .
\end{aligned}
$$

The last equation explicitly demonstrates the aforementioned cancellations between the derivatives of $E_{<}$and $E_{>}$. The value of the delta-function product average is determined by properties of typical configurations. Hence, the low probability of having unusually shallow local minima is entirely due to the first factor on the left-hand side of Eq. (79) and so $P(\alpha) \propto|\alpha|$. In particular, $P \propto \epsilon$ in the case of interest (78). But this is not yet the end of the story. Following Aleiner and Ruzin 34 we argue that the probability of having a shallow global minimum is additionally suppressed. Indeed, if the coefficient $\beta$ in front of the the cubic term in Eq. $(77)$ is too large, $E(X)$ would have a second minimum which is deeper than that at the ground state $X=0$. To avoid that $|\beta|$ must not exceed $\gamma \sqrt{\alpha} \propto \sqrt{\epsilon}$. The typical dependence of the resultant $E(X)$ is illustrated in Fig. \&.

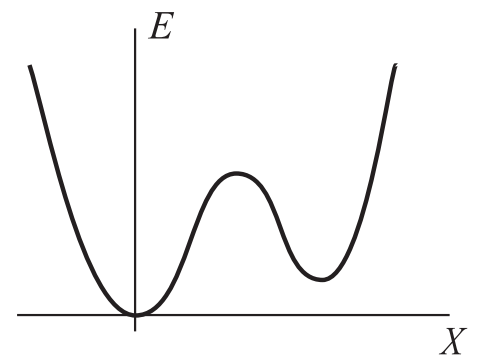

FIG. 8. Energy of the system as a function of the collective coordinate $X$.

The total probability density of the optimal fluctuation is therefore proportional to $\epsilon \cdot \sqrt{\epsilon}=\epsilon^{s}$ with $s=3 / 2$, as we claimed.

It is very likely that $s=3 / 2$ is in fact the general result independent of the number of dimensions because the optimal fluctuation would always have only one soft direction, i.e., the system is essentially one-dimensional 34

Finally, let us compare our results with those in the literature. One group of works, by Feigelman et al 33 has been already mentioned before. We borrowed from them the idea of splitting the system into two statistically independent parts. The major mistake of these authors is overlooking the possibility of frustrations in the system. In other words, they did not realize that it is not necessary to require that both $E_{<}$and $E_{>}$have a small second derivative with respect to $u$ when only their sum $E_{<}+E_{>}$needs to be so. Another point of disagreement between us and them is the size of the localized phonons. Feigelman et al. take for granted that it is of the order of $\sqrt{\mu / \epsilon}$, while we gave an argument that it should be $R_{c}$, i.e., much smaller.

In another large group of papers, Refs. 9, 10, 19, 35, $\nu_{\omega}(\omega) \propto \omega^{2}$ for the density of states, and a similar dependence, $\operatorname{Re} \sigma_{x x}(\omega) \propto \omega^{2}$, for the conductivity were calculated. To facilitate the comparison we should point out that the conventionally defined phonon density of states $\nu_{\omega}(\omega)$ is related to our $\nu(\epsilon)$ by

$$
\nu_{\omega}(\omega)=2 \nu\left(\rho \omega^{2}\right) \rho \omega .
$$

Hence, our result is $\operatorname{Re} \sigma_{x x}(\omega) \propto \nu_{\omega}(\omega) \propto \omega^{4}$, same as in Refs. 34 (see also Ref. 36). All the papers in the sec- 
ond group are explicitly or implicitly based on the SCBA (see Appendix B for more details). The second power of $\omega$ can be traced to the unphysical square-root singularity $\nu(\epsilon) \propto\left|\epsilon-\epsilon_{\mathrm{th}}\right|^{1 / 2}$ near a band edge $\epsilon_{\mathrm{th}}\left(\epsilon_{\mathrm{th}}=0\right.$ in our case), which is a well-known basic flaw of the SCBA.20 Note however that even within the SCBA $\nu(\epsilon)$ conforms to the generic form (76), except it corresponds to $s=1 / 2$ instead of what we believe is the correct result, $s=3 / 2$.

\section{QUANTUM AND THERMAL EFFECTS}

So far we have neglected any effects of quantum nature or due to a finite temperature. Some of them will be addressed in this section but their systematic, in-depth treatment is deferred for future work.

Let us begin with noting that the electrons of the pinned WC constantly fluctuate around their equilibrium positions. The typical size of such fluctuations is easy to find. For example, in strong magnetic fields and at low temperatures $\left(k_{B} T \ll \hbar \omega_{c}\right)$ where all the electrons are confined to the lowest Landau level, we have

$$
\begin{aligned}
& \left\langle\mathbf{u}^{2}\right\rangle=l_{B}^{2}+\frac{\hbar}{\rho \omega_{c}} \int_{\mathbf{q}} \operatorname{coth} \frac{\hbar \Omega(\mathbf{q})}{2 k_{B} T} \\
& \quad \times \frac{1}{\rho \omega_{c} \Omega(\mathbf{q})}\left[\Pi_{0}+\frac{\mu(\mathbf{q})+\lambda(\mathbf{q})}{2} q^{2}\right], \\
& \Omega(\mathbf{q})=\left(\rho \omega_{c}\right)^{-1} \sqrt{\left.\left[\Pi_{0}+\mu(\mathbf{q}) q^{2}\right)\right]\left[\Pi_{0}+\lambda(\mathbf{q}) q^{2}\right]} .
\end{aligned}
$$

This formula can be used away from the thermal or quantum melting transitions, where the phenomenological criterion $\left\langle\mathbf{u}^{2}\right\rangle^{1 / 2}<\varepsilon a$ is satisfied. Here $\varepsilon \sim 0.2$ is the Lindenmann parameter 8 Incidentally, at $T=\Pi_{0}=0$, Eq. (81) gives the variance of the electren fluctuations in the correlated WC of Lam and Girvin. 37

Let us focus on the range of temperatures $k_{B} T \ll \hbar \omega_{B}$ where $\omega_{B} \sim 4 \pi \mu / m_{e} \omega_{c}$ is the magnetophonon bandwidth (usually, $\hbar \omega_{B} / k_{B} \sim 2 \mathrm{~K}$ ). At such temperatures $\left\langle\mathbf{u}^{2}\right\rangle^{1 / 2}$ is totally dominated by quantum fluctuations and is of the order of $l_{B}$. We expect that as long as $\left\langle\mathbf{u}^{2}\right\rangle^{1 / 2}$ is smaller than the correlation length of the pinning potential $\xi$, which is the smallest length scale in the classical theory, all the results obtained in the previous sections acquire at most minor corrections.

Recently Fertig 18 and Chitra et al. 19 studied the opposite limit, $\xi \gg l_{B}$, and found a novel dependence of the pinning frequency $\omega_{p}$ on the magnetic field, although they disagree with each other on the functional form of such a dependence. To sort things out we offer a different perspective on this question. Recall that the classical theory predicts the $1 / B$-behavior, 10

$$
\omega_{p} \sim \frac{C(0) c}{e \mu \xi^{4} B},
$$

which follows from Eqs. (14), (35), and (39). We propose that a reasonably accurate estimate for $\omega_{p}$ in the regime $\xi \ll l_{B}$ can be obtained within a quasiclassical approximation. The electrons are visualized as compact wavepackets whose centers of gravity perform classical motion, while the quantum effects are presumed to be contained in the form-factor of the wavepakets. This type of quantum effects amount to replacing the bare pinning potential $U(\mathbf{r})$ by its convolution with the form-factor $F_{e}(\mathbf{r})$ of the wavepackets. For small fluctuations $l_{B} \ll a$ the appropriate form-factor is Gaussian, $F_{e}(\mathbf{r})=\left(\pi\left\langle\mathbf{u}^{2}\right\rangle\right)^{-1} \exp \left(-r^{2} /\left\langle\mathbf{u}^{2}\right\rangle\right)$. Upon the convolution, we obtain an effective random potential, with the correlation length $l_{B}$ and variance $C(0) \xi^{2} / l_{B}^{2}$. In view of such modifications, Eq. (83) transforms into

$$
\omega_{p} \sim \frac{e^{2}}{\mu \hbar^{3} c^{2}} C(0) \xi^{2} B^{2}, \quad \xi<l_{B}
$$

The quasiclassical approximation is certainly not exact; nonetheless, when used away from the quantum Hall fractions, at sufficiently low $T$, and for robust quantities such as $\omega_{p}$, it should be qualitatively correct.

The linear in $B$-dependence of $\omega_{p}$ derived by Fertig [see Eq. (44) in Ref. 18 is at odds with the $B^{2}$-dependence found above [Eq. (84)] and in Ref. 19. Although Fertig consider a Poissonian disorder (potential wells or "pits" of size $s<l_{B}$, energy $\Delta V$, and the areal density $n_{i}<1 / \pi l_{B}^{2}$ ) rather than the Gaussian one, the real source of the discrepancy is his ascertion that the elastic distortion at the Larkin length scale is of the order of $n_{i}^{-1 / 2}$. Instead, $l_{B}$ should be used. Upon this correction, the formula for $\omega_{p}$ acquires the above form (84) with $C(0) \xi^{2}$ replaced by $n_{i} s^{4} \Delta V^{2}$.

The finite amplitude of the quantum fluctuations has another important repercussion: nonvanishing highorder in $\mathbf{u}$ terms in the pinning energy. As a result, the absorption line acquires extra broadening even at $T=0$. It originates from finite widths $\Gamma_{n}$ of the energy levels $n=1,2, \ldots$ of the localized magnetophonon oscillators (the ground state $n=0$ remains unbroadened). At zero $T$ only the transitions between $n=0$ and $n=1$ levels contribute to the absorption. The level width $\Gamma_{1}$ of the $n=1$ state is determined by the decay of the given magnetophonon into two other magnetophonon excitations of lower frequencies, $\hbar \omega_{1} \rightarrow \hbar \omega_{2}+\hbar \omega_{3}$ (see Fig. 9).

Such a process is always possible in a truly random system, the necessary three-phonon matrix elements,

$$
M_{\alpha \beta \gamma}(\mathbf{r})=-n_{e} \sum_{\mathbf{K}} K_{\alpha} K_{\beta} K_{\gamma} U(\mathbf{r}) \sin \mathbf{K}\left[\mathbf{r}-\mathbf{u}^{(0)}(\mathbf{r})\right],
$$

being provided by the cubic anharmonicities. The corresponding contribution $\operatorname{Im} \Pi_{3}$ to the imaginary part of the self-energy can be obtained by evaluating the diagram in Fig. 9 ,

$$
\operatorname{Im} \Pi_{3}(\mathbf{q}, \omega)=-\frac{2 \hbar}{L^{2}} \int_{\mathbf{k}} \int_{\mathbf{k}^{\prime}}\left\langle\tilde{M}_{\alpha \mu \sigma} \tilde{M}_{\beta \nu \tau}\right\rangle \int \frac{d \omega^{\prime}}{2 \pi}
$$




$$
\begin{aligned}
& \times\left[n_{B}\left(\omega^{\prime}\right)+n_{B}\left(\omega-\omega^{\prime}\right)+1\right] \\
& \times \operatorname{Im} D_{\mu \nu}\left(\mathbf{k}, \omega^{\prime}\right) \operatorname{Im} D_{\sigma \tau}\left(\mathbf{k}^{\prime}, \omega-\omega^{\prime}\right), \\
n_{B}(\omega) & =\frac{1}{\exp \left(\hbar \omega / k_{B} T\right)-1} .
\end{aligned}
$$

At $k_{B} T \ll \hbar \omega_{p}$ only the frequency interval $0<\omega^{\prime}<\omega$ contributes to the integral, which leads us to the estimate

$$
\operatorname{Im} \Pi_{3}\left(\omega_{p}\right) \sim \operatorname{Im} \Pi\left(\omega_{p} / 2\right) \frac{l_{B}^{2}}{\xi^{2}} \frac{a^{2}}{R_{c}^{2}} \frac{\left|\operatorname{Im} \Pi\left(\omega_{p} / 2\right)\right|}{\Pi_{0}} .
$$

This formula is derived assuming that $\xi \gg l_{B}$; otherwise, in the spirit of the quasiclassical approximation, we have to replace $\xi$ by $l_{B}$. Even in that case $\operatorname{Im} \Pi_{3}\left(\omega_{p}\right)$ is smaller than the previously found $\operatorname{Im} \Pi\left(\omega_{p}\right)$ by a large factor $\sim\left(R_{c} / a\right)^{2}(\lambda / \mu)^{s}$. At higher temperatures, the anharmonic effects are enhanced by a factor of $k_{B} T / \hbar \omega_{p}$ originating from the Bose distribution functions $n_{B}$ in Eq. (85). They should start to affect the linewidth when $k_{B} T \sim\left(R_{c} / a\right)^{7 / 2} \hbar \omega_{p}$ (cf. Ref. 38).
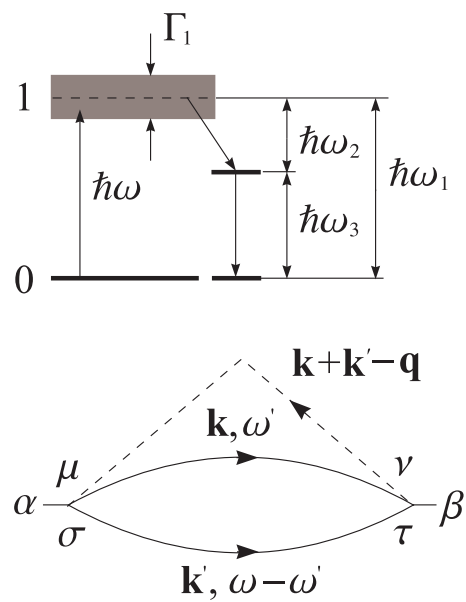

FIG. 9. Top: low-temperature absorption in a system with energy levels broadened by anharmonicities. Bottom: the diagram used for estimating $\Gamma_{1}$.

A word of caution is in order. The diagram in Fig. 9 does not contain vertex corrections, which contain information about, e.g., the soft modes. The soft modes are crucial for the dynamical response in strong magnetic fields and at the same time are potential sources of much larger anharmonicities. Thus, the response of a weakly pinned quantum WC may prove to be more nontrivial.

Concluding this section, we would like to re-emphasize that for the problem studied in the main part of the paper (zero-temperature linear-response of a classical WC), the anharmonicities are not important.

\section{COMPARISON WITH EXPERIMENT}

In this section we will thempt to analyze the most recent experimental data 16.10 in the light of our under- standing of the pinning mode. Let us first discuss the position of the pinning resonance.

From Eqs. (83) and (84) we see that depending on the type of disorder, $\omega_{p}$ can either decrease as $1 / B$ or increase as $B^{2}$ when the magnetic field increases.19 Weak magnetiffield dependence of $\omega_{p}$ found in the experiments16.17 leads us to conclude that $\xi$ remains of the order of $l_{B}$ in the limited range $10-15 \mathrm{~T}$ of the magnetic fields where the pinning mode was detected, i.e., $\xi \approx l_{B} \approx 75 \AA$. The source of such a short-range random potential is likely to be the roughness of the heterostructure interface as suggested by Fertig. 18 The experimental value of $\omega_{p} \approx 8 \times 10^{9} \mathrm{~s}^{-1}$ for $n_{e}=5.4 \times 10^{11} \mathrm{~cm}^{-2}$ can then be reproduced with reasonable values of fitting parameters (see Sec. VI) $s=30 \AA, \Delta V=4.3 \mathrm{~K}$, and $n_{i}=4 \times 10^{11} \mathrm{~cm}^{-2}$. Unfortunately, the disorder parameters are poorly known, and so we have to use $\omega_{p}$ to estimate the disorder characteristics, not the other way around. For example, we can solve Eq. (84) for the rms amplitude $\sqrt{C(0)}$ of the pinning potential, which gives a value of the order $0.2 \mathrm{~K}$.

Let us now discuss the density dependence of $\omega_{p}$. Explicitly, it enters Eq. (84) only through the shear modulus $\mu\left(n_{e}\right)$. The latter should behave as $\mu \propto n_{e}^{3 / 2}$ (see Sec. III) away from the quantum Hall fractions, e.g., the $\frac{1}{3}$-filling. If $C(0)$ is $n_{e}$-independent, we therefore expect $\omega_{p} \propto n_{e}^{-3 / 2}$. Such a dependence was indeed observed by $\mathrm{Li}$ et al.16 for the concentration of holes ( $p$-type samples were used) in the range $3-5 \times 10^{10} \mathrm{~cm}^{-2}$, which corresponds to the filling factor range $0.1-0.16$. At lower concentrations the pinning frequency continued growing as $n_{e}$ decreased but less rapidly. This comes presumably from the fact that the pinning is no longer weak at such low $n_{e}$ : one can verify that $R_{c}$ is approximately $6 a$ at the highest densities, but approaches $a$ (the lattice constant) at the lowest densities used in Ref. 16. (In Ref. 17 the density dependence of $\omega_{p}$ was not investigated).

The relationships among the empirical values of $\omega_{p}$ and $\Delta \omega_{p}$ and the experimental parameters $\left(n_{e}\right.$ and $\left.B\right)$ provide another means to test the agreement between the theory and the experiment. To this end we rewrite Eq. (3) (with $s=3 / 2$ ) in the following way:

$$
Q \equiv \frac{\omega_{p}}{\Delta \omega_{p}} \sim\left[\left(26 \frac{e c}{\kappa} \frac{n_{e}^{3 / 2}}{B f_{p k}}\right)^{1 / 2}-5\right]^{3 / 2},
$$

where $f_{p k}=\omega_{p} /(2 \pi)$ is the pinning frequency in cycles per second. The largest quality factor $Q \approx 8$ reported by Li et al. was achieved for $n_{e}=5.4 \times 10^{10} \mathrm{~cm}^{-2}, B=13 \mathrm{~T}$, where $f_{p k}$ was measured to be $1.4 \mathrm{GHz}$. In Ref. $17 Q \approx 45$ was found for comparable $n_{e}, B$, and $f_{p k}$. Our theoretical estimate from Eq. (88) is $Q \approx 260$ and far exceeds both. We should point out, however, that specific details of the experimental setup become important for observability of sych a high $Q$. Henceforth we focus on the work of $\mathrm{Li}$ et al.16 since most of their data is available in the published record. 
Because of the finite distance $(30 \mu \mathrm{m})$ between the plates of the coplanar microwave waveguide (see Fig. 10), these were not the ideal $\mathbf{q}=0$ measurements. We estimate that the spread of q's imposes un upper bound of about 30 for the largest observable $Q$. The remaining discrepancy seems to be rooted in finite-temperature and to a lesser degree finite incident power effects ignored in the derivation of Eq. (88). In the experiments, the linewidth was decreasing roughly linearly as the temperature varied from $T=200 \mathrm{mK}$ to $T=50 \mathrm{mK}$ with other parameters held fixed. Below $50 \mathrm{mK}$ the linewidth appeared to reach saturation, but only at the nominal level of incident microwave power $(80 \mathrm{pW})$. When lower power levels were used, the line continued sharpening up. At the lowest experimental temperature of $25 \mathrm{mK}$, the linewidth did not show any signs of saturation (this time as a function of power) even upon ten-fold input power reduction, which was near the sensitivity limit.
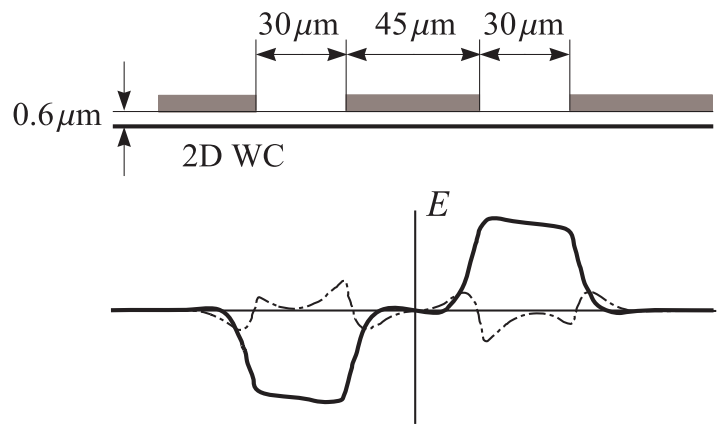

FIG. 10. Top: schematics of the microwave setup used by $\mathrm{Li}$ et al. The transmission line is shaded, the $2 \mathrm{D}$ electron (or more precisely, hole) gas is represented by the thick horizontal line underneath (adapted from Ref. 16). Bottom: typical form of the electric field distribution in the plane of the WC. 39 Solid line is the signal in phase with the source, dashed-dotted line is the signal in quadrature.

Several sources of the thermal broadening can be envisioned: (a) thermally excited single-particle excitations of the WC, 12 (b) phonons of the host semiconductor, (c) the anharmonisms of the collective modes of the pinned WC, and perhaps some others. 18

The classical activation energy of vacancies and interstitials is of the order of $0.5 \mathrm{~K}$ at the densities studied, and mechanism (a) should be frozen out at $25 \mathrm{mK}$. Furthermore, for the WC of low density the bandwidth of such excitations is very narrow and so they are easily localized by the random potential.

The weak electron-phonon coupling in GaAs and small phonon phase space at the frequencies involved $\left(\omega_{p}\right)$ are likely to render the mechanism (b) inefficient.

The preliminary estimate of the broadening due to mechanism (c) was given in the previous section. It is too small to explain the experimental observations. We speculate that stronger anharmonic effects and a better agreement with the experiments may be obtained if one properly accounts for untypically large anharmonicities at the locations of the soft modes, which as we showed in this paper, play a very important role in the response.

It is also quite possible that the actual pinning potential is more complicated than the one studied here. In reality, it can be due to a combination of the interface roughness 18 and dilute residual ions in the vicinity of the WC plane 40 In this case $\omega_{p}$ may be determined by the former, while $Q$ could be limited by the latter. Such more complicated models as well as the dynamic response of a pinned quantum $\mathrm{WC}$ are interesting subjects awaiting further investigation.

It would be also interesting to investigate if the discussed phenomena appear in the conventional chargedensity waves (see Sec. [I). These materials are threedimensional, and some important modifications of the present theory may be needed. There are other complications such as an extra dissipation due to uncondensed quasiparticles but those can be suppressed by lowering the temperature. In any case, it would be remarkable if narrow pinning modes could be produced in these materials simply by applying a sufficiently strong magnetic field.

Our theory should literally apply to the pinning mode of a WC formed by electrons on solid hydrogen (see the second book cited under Ref. 8). We hope that this paper will encourage further experiments on these or other numerous systems where the pinning manifests itself.

\section{ACKNOWLEDGMENTS}

This research is supported by US Department of Energy Grant No. DE-FG02-90ER40542 and NSF Grant No. DMR-9802468. We thank R. Chitra, Mark Dykman, Lloyd Engel, Herb Fertig, Gabi Kotliar, Chi-Chun Li, Leonid Pryadko, Misha Raikh, Dan Tsui, and Valerii Vinokur for useful discussions. M. M. F. is grateful to Tito Williams for providng a copy of Ref. 17 .

\section{APPENDIX A: MODEL CALCULATION FOR L-L SCATTERING}

In order to evaluate the importance of the L-L scattering channel, let us consider the model action

$$
\begin{aligned}
A_{L}(\epsilon) & =\frac{1}{2} \int_{\mathbf{q}} u_{L}^{*}(\mathbf{q})\left[H_{L}^{0}(\mathbf{q})-\epsilon\right] u_{L}(\mathbf{q}) \\
& +\frac{1}{2} \int_{\mathbf{r}} u_{L}^{*}(\mathbf{r}) S_{L}(\mathbf{r}) u_{L}(\mathbf{r}) .
\end{aligned}
$$

where $S_{L}$ is a local operator of a Gaussian white-noise type with parameters

$$
\left\langle S_{L}\right\rangle=\Pi_{0}, \quad\left\langle S_{L}\left(\mathbf{r}_{1}\right) S_{L}\left(\mathbf{r}_{2}\right)\right\rangle=\Pi_{0}^{2}-V^{\prime \prime}(0) \delta\left(\mathbf{r}_{1}-\mathbf{r}_{2}\right) .
$$


Our objective is to calculate the disorder-averaged Green's function

$$
D_{L}(\mathbf{q}, \epsilon) \equiv \frac{i}{L^{2}}\left\langle\frac{\int \mathcal{D} u_{L} \mathcal{D} u_{L}^{*} u_{L}(\mathbf{q}) u_{L}^{*}(\mathbf{q}) e^{-i A_{L}(\epsilon)}}{\int \mathcal{D} u_{L} \mathcal{D} u_{L}^{*} e^{-i A_{L}(\epsilon)}}\right\rangle,
$$

where an infinitesimal imaginary correction is assumed to be included via $\epsilon \rightarrow \epsilon+i 0$ to make the integrals convergent.

Using the "supersymmetry" technique $41 D_{L}$ can be represented in the form

$$
D_{L}=\frac{i}{L^{2}}\left\langle\int \mathcal{D} \phi \mathcal{D} \phi^{*} u_{L} u_{L}^{*} e^{-i A_{s}[\phi]}\right\rangle .
$$

where $\boldsymbol{\phi}_{i}^{\dagger}=\left[u_{L}^{*} v_{L}^{*}\right]$ is a supervector, $v_{L}(\mathbf{q}, \omega)$ is an auxillary fermionic field, and $A_{s}[\boldsymbol{\phi}]$ is the sypersymmetric Eucledian action

$$
\begin{aligned}
A_{s} & =\frac{1}{2} \int_{\mathbf{q}} \phi^{\dagger}(\mathbf{q})\left(Y q+\Pi_{0}-\epsilon\right) \phi(\mathbf{q}) \\
& +\frac{i}{8} V^{\prime \prime}(0) \int_{\mathbf{r}}\left[\phi^{\dagger}(\mathbf{r}) \phi(\mathbf{r})\right]^{2} .
\end{aligned}
$$

Note that the coefficient in front of the quartic term is imaginary, which makes the action non-Hermitian. The sign of the imaginary part is such that excitations above the vacuum state $\phi \equiv 0$ decay after travelling a certain distance. This distance is simply the phonon mean free path.

It is easy to establish some of the properties of the propagator $D_{L}$ right away. Since the field $\phi$ is massive for $\epsilon \lesssim \Pi_{0}$ at the tree level, the imaginary part of the propagator $D_{L}$ is small at such $\epsilon$. However, $\operatorname{Im} D_{L}$ should grow rapidly as soon as $\epsilon$ crosses the $\epsilon=\Pi_{0}$ threshold. Below we are going to verify this by explicit calculations. Although our methods do not work in the immediate vicinity of $\epsilon=\Pi_{0}$, the expressions obtained for $\epsilon<\Pi_{0}$ and $\epsilon>\Pi_{0}$ can be smoothly matched onto each other.

Rescaling the fields in Eq. (A2) by $\sqrt{2 / Y}$, we obtain the action with the Lagrangian

$$
\mathcal{L}=\boldsymbol{\phi}^{\dagger} \hat{K} \boldsymbol{\phi}+\frac{M}{2} \boldsymbol{\phi}^{\dagger} \boldsymbol{\phi}-i \frac{g}{4}\left(\boldsymbol{\phi}^{\dagger} \boldsymbol{\phi}\right)^{2}
$$

where $\hat{K}$ is the operator, which corresponds to multiplication by $|\mathbf{q}|$ in the momentum representation, $M=$ $2\left(\Pi_{0}-\epsilon\right) / Y$ plays the role of mass, and $g \equiv-2 V^{\prime \prime}(0) / Y^{2}$ is the bare coupling constant. The coupling constant is dimensionless, and it is possible to show that the field theory is renormalizable. Let us derive the Wilsonstyle renormalization group ( $R G$ ) equations for the renormalized parameters $g_{R}$ and $M_{R}$. As usual, it is done by successive integrations over narrow momentum shells $\Lambda(l+\delta l)<q<\Lambda(l)$ where $\Lambda(l)=\Lambda_{c} e^{-l}$ and $\Lambda_{c} \sim 1 / a$ are the running and the bare high-momentum cutoffs, respectively.
Consider the effect of such an integration on $g_{R}$ first. Initially, $g_{R}$ remains small and we need to take into account only the three lowest order in $g_{R}$ diagrams (see Fig. 11a) to find

$$
\begin{aligned}
& g_{R}(l+\delta l)=g_{R}(l)+\frac{2}{3} g_{R}^{2} \int_{e^{-\delta l} \Lambda<q<\Lambda} \frac{d^{2} q}{(2 \pi)^{2}} \frac{1}{q+M_{R}} \\
& \times\left[\frac{1}{|\mathbf{q}+\mathbf{s}|+M_{R}}+(\mathbf{s} \rightarrow \mathbf{t})+(\mathbf{s} \rightarrow \mathbf{u})\right],
\end{aligned}
$$

where $S=\mathbf{s}^{2}=\left(\mathbf{q}_{1}-\mathbf{q}_{2}\right)^{2}, T=\mathbf{t}^{2}=\left(\mathbf{q}_{1}-\mathbf{q}_{3}\right)^{2}$, and $U=\mathbf{u}^{2}=\left(\mathbf{q}_{1}-\mathbf{q}_{4}\right)^{2}$ are the Mandelstam variables with $\mathbf{q}_{i}, i=1, \ldots, 4$ being the incoming momenta. Similarly, the tadpole diagram shown in Fig. 11b determines the variation of the renormalized mass $M_{R}$,

$$
M_{R}(l+\delta l) \simeq M_{R}(l)-\frac{g_{R}}{2} \int_{e^{-\delta l}} \frac{d^{2} q}{(2 \pi)^{2}} \frac{1}{|\mathbf{q}|+M_{R}} .
$$

(a)

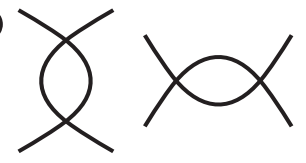

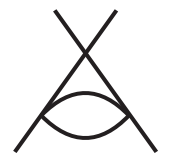

(b)

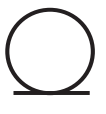

FIG. 11. Diagrams for the (a) coupling constant and (b) mass term.

In addition to $g_{R}$ and $M_{R}$, the kinetic term $\hat{K}$ also gets renormalized. We will neglect such an effect because it is of higher (second) order in $g_{R}$. Consequently, the spectral function $\operatorname{Im} D_{L}$ is determined just by the mass,

$$
\operatorname{Im} D_{L}(q, \epsilon)=\frac{2}{Y} \frac{\operatorname{Im} M_{R}}{\left(q+\operatorname{Re} M_{R}\right)^{2}+\left(\operatorname{Im} M_{R}\right)^{2}} .
$$

In this equation $M_{R}=M_{R}(q, \epsilon)$ stands for the renormalized mass at such large negative $l$ where the RG flow eventually stops either because $q \sim \Lambda(l)$ or because $\left|M_{R}\right| \sim \Lambda(l)$. Below we consider exclusively $q=0$ case.

From Eq. (A4) we find that in the limit $\left|\mathbf{q}_{i}\right|,\left|M_{R}\right| \ll \Lambda$ function $\beta \equiv g_{R} / \partial l$ depends only on $g_{R}$,

$$
\beta=\frac{1}{\pi} g_{R}^{2},
$$

and so the RG flow equation is easy to solve:

$$
g_{R}=\frac{\pi}{l_{L}-l}, \quad l_{L} \equiv \frac{\pi}{g} .
$$

At $l \approx l_{L}-\pi, g_{R}$ becomes of the order of one, and the theory enters the strong coupling regime. The corresponding spatial scale is $L_{L-L} \sim \Lambda_{c}^{-1} \exp (\pi / g)$. Since the strength of coupling maps to the strength of the effective disorder in the original formulation, $L_{L-L}$ has the meaning of the L-phonon localization length (due to L-L scattering). Note that $g_{R}$ seems to exhibit a divergence (Landau pole) 
at $l=l_{L}$. This is an artifact of the one-loop RG. We expect that instead $g_{R}$ flows towards its fixed point value of the order of one.

The other scaling equation, for $M_{R}$, can be deduced from Eq. (A5),

$$
\gamma_{M} \equiv \frac{\partial M_{R}}{\partial l}=-\frac{g_{R}(l)}{4 \pi} \frac{\Lambda^{2}(l)}{\Lambda(l)+M_{R}-i \delta} .
$$

The solution cannot be not expressed in elementary functions but we can describe its asymptotics.

1. $(g / 4 \pi) \Lambda_{c}=M_{*} \ll|M| \ll \Lambda_{c}$.

Here the renormalization of the coupling constant is not important because the RG flow is effectively cut off at $l \sim \ln \left(\Lambda_{c} /\left|M_{R}\right|\right)$ before $g_{R}$ manages to get large. Equation (A9) reduces to the SCBA equation

$$
M_{R}=M-\frac{g}{4 \pi} \int_{\Lambda}^{\Lambda_{c}} \frac{d q q}{q+M_{R}-i \delta},
$$

with the approximate solution

$$
\begin{aligned}
& \operatorname{Re} M_{R} \simeq M-M_{*}, \\
& \operatorname{Im} M_{R} \simeq(g / 4) \Theta\left(-\operatorname{Re} M_{R}\right) \operatorname{Re} M_{R},
\end{aligned}
$$

where $\Theta(x)$ is the Heaviside step-function. A word of caution is in order here. The imaginary part of $M_{R}$ vanishes at positive $\operatorname{Re} M_{R}$ only at the level of the one-loop RG. Nonperturbative methods indicate that $\operatorname{Im} M_{R}$ is nonzero albeit exponentially small (see below).

2. $L_{L-L}^{-1} \ll\left|M-M_{*}\right| \ll M_{*}$.

Now instead of Eq. A11 we have

$$
\begin{aligned}
& \operatorname{Im} M_{R} \simeq \frac{1}{4} \frac{\pi}{l_{L}-l_{*}} \Theta\left(-\operatorname{Re} M_{R}\right) \operatorname{Re} M_{R} \\
& \operatorname{Re} M_{R} \simeq\left(\frac{l_{L}}{l_{L}-l_{*}}\right)^{1 / 4}\left(M-M_{*}\right), \\
& l_{*}=\ln \left(-\frac{\Lambda_{c}}{\operatorname{Re} M_{R}}\right) .
\end{aligned}
$$

The above comment about small but nonzero $\operatorname{Im} M_{R}$ at $\operatorname{Re} M_{R}>0$ applies here as well.

3. $\left|M-M_{*}\right| \lesssim L_{L-L}^{-1}$.

Since the one-loop RG cannot be trusted beyong the point $l=l_{L}-\pi$, we choose to terminate the RG procedure at this stage. Unfortunately, this leaves us with a theory which (a) does not have any small parameters and (b) includes not only the original quartic but also higher order interaction terms generated by the RG. However, on physical grounds we can expect that in this, essentially massless, limit, the mass scale must be set by the running cutoff $\Lambda\left(l_{L}\right)$, i.e.,

$$
\operatorname{Im} M_{R} \sim \Lambda_{c} g^{-1 / 4} e^{-\pi / g} .
$$

This can be compared with the solution of the SCBA equation $(\mathrm{A} 10)$ in the massless limit $\left(\operatorname{Re} M_{R}=0\right)$,

$$
\operatorname{Im} M_{R}^{\mathrm{SCBA}} \sim \Lambda_{c} e^{-4 \pi / g} .
$$

Clearly, the SCBA misses the factor of four in the exponential.

As mentioned above, RG-enhanced perturbation theory formulas A11b) and A12a do not work at low energies. Nonvanishing $\operatorname{Im} M_{R}$ can be detected using the nonperturbative method of constrained instantons. 39 The details of the calculation will be presented elsewhere. Here we only quote the result,

$$
\operatorname{Im} M_{R} \simeq \frac{4 \pi e \sqrt{6 \pi}}{9 g_{R}^{2}} \ln \left(\frac{\Lambda_{c} g_{R}}{\operatorname{Re} M_{R}}\right) \Lambda_{c} e^{-\pi / g},
$$

which is valid for $\operatorname{Re} M_{R} \gg 1 / L_{L-L}, g_{R} \ll 1$. Equations (A13) and (A15) match for $\left|\operatorname{Re} M_{R}\right| \sim 1 / L_{L-L}$. Therefore, we expect that the spectral function $\operatorname{Im} D_{L}$ exhibits a quasi-Lorentzian peak centered at $\epsilon_{p}=Y M_{*} \simeq$ $\Pi_{0}$ of exponentially small width

$$
\Delta \epsilon_{p}=\frac{Y}{2} \operatorname{Im} M_{R}\left(0, \epsilon_{p}\right) \sim \Lambda_{c} Y g^{-\omega} e^{-\pi / g} .
$$

\section{APPENDIX B: DYNAMICAL RESPONSE WITHIN THE SCBA}

Within the SCBA the self-energy $\Pi_{\alpha \beta}(\omega, q)$ is diagonal, $\Pi_{\alpha \beta}(\omega, q)=\delta_{\alpha \beta} \Pi$ and weakly $q$-dependent for $q a \ll 1$. $\Pi$ is to be found from the self-consistency equation (37) which we reproduce here for convenience,

$$
\Pi(\omega)=S_{0}+V^{\prime \prime}(0) \int_{\mathbf{k}} \operatorname{tr} D(\mathbf{k}, \omega) .
$$

As discussed in Secs. III and IV, the SCBA applies only at relatively high frequencies. Moreover, in strong magnetic fields and near the pinning frequency $\omega \sim \omega_{p}$, it is not even qualitatively correct. One may ask why we bother elaborating on this faulty approximation scheme here. The reason is as follows. We discovered that as far as the dynamical response is eqncerned, all alternative theories suggested so far $10,12,18,19$ are merely different forms of the SCBA. Thus, we deemed that it would be helpful to expose their interrelationship and correct a few calculational errors.

Let us briefly summarize the resultsobtained by previous authors. Fukuyama and Lee 10 concluded that $\Delta \omega_{p} \sim \omega_{p}$ but did not present the detajls of the calculation. Perturbative analysis of Fertig 42 yields an exponentially small $\Delta \omega_{p} / \omega_{p}$ (for weak disorder). Gaussian 
variational replica method (GVM) of Chitra et al 19 reduces to the SCBA at nonzero frequencies, and when analyzed further, predicts a power law dependence of $\Delta \omega_{p}$ on the disorder strength. Finally, the SCBA analysis in the Appendix of Ref. 12 leads to yet another dependence. One reason for such a disarray of conflicting results is the extreme sensitivity of the solution $\Pi(\omega)$ of Eq. (B1) to the precise value of the dimensionless parameter $C \equiv-V^{\prime \prime}(0) /\left(4 \pi \mu \Pi_{0}\right)$, where $\Pi_{0} \equiv \Pi(0)$. In principle, $C$ is fully determined by $S_{0}$, but $S_{0}$ is known only approximately. A strong partial cancellation of $S_{0}$ by the second term in Eq. (B1) leaves us only the order of magnitude estimate $C \sim 1$. One could argue, as Fukuyama and Lee did in analogous situation, 9 that $C=1$ is the "best" choice because others lead to various physical absurdities. For example, if $C>1$, then $\Pi(\omega)$ acquires a finite imaginary part at complex $\omega$, which means that the ground state is unstable; if $C<1$, then $\Pi(\omega)$ has an imaginary part only above some threshold frequency $\omega_{\text {th }}>0$, i.e., the phonon spectrum has a gap, which also appears to be unphysical. The truth is, of course, that the SCBA is simply unable to correctly describe the properties of the $\omega \rightarrow 0$ tail; therefore, it cannot be relied upon for selecting a "good" value of $C$. More sensible approach is to investigate a certain range of $C$ around unity, hoping that certain quantities depend on $C$ only weakly. Alas, the linewidth $\Delta \omega_{p}$ is not one of such quantities.

We found that the results of Chitra et al. are recovered for the "Fukuyama-Lee choice," $C=1$. (Therefore, these two groups of authors should have obtained the same results). A slight reduction of $C$ from unity is sufficient to cross over to very different predictions of Fertig. Let us now demonstrate this in more detail.

Using the definition of $C$ and Eq. (B1), we obtain

$$
\Pi(\omega)-\Pi_{0}=2 \mu C \Pi_{0} \int_{0}^{\infty} d k k\left[D_{T}(k, 0)-D_{T}(k, \omega)\right] .
$$

Let us now describe the solution $\Pi(\omega)$ of this equation for different $C$ in the weak pinning regime $R_{c} \gg a$, which corresponds to the inequality $\alpha \equiv \sqrt{\Pi_{0} \mu} / Y=\mu / \lambda \ll 1$.

1. $0<1-C \lesssim \alpha$.

In this case

$$
\Pi(\omega) \simeq C \Pi_{0}+i \Pi_{0} \sqrt{\left(\omega / \tilde{\omega}_{p}\right)^{2}-(1-C)^{2}},
$$

where $\tilde{\omega}_{p}=\omega_{p} /(\pi \alpha)^{1 / 2}$ and $\omega_{p}=\Pi_{0} / \rho \omega_{c}$. As one can see, the threshold frequency is $\omega_{\text {th }}=(1-C) \tilde{\omega}_{p}$. For $C=1 \mathrm{Eq}$. (B3) coincides with that of Ref. 19. Strictly speaking, Eq. (B3) describes the solution of Eq. (B2) only for $\omega \ll \omega_{p}$. Near the resonance, $\omega \sim \omega_{p}$, it is off by a numerical factor of the order of unity. In principle, this factor can also be calculated. We limit ourselves to the order of magnitude estimate $\operatorname{Im} \Pi\left(\omega_{p}\right) \sim \alpha^{1 / 2} \Pi_{0}$, which leads to $\Delta \omega_{p} / \omega_{p} \sim \alpha^{1 / 2}=(\mu / \lambda)^{1 / 2}$. Although this result is of the same form as Eq. (66), with $s=1 / 2$, we believe that $s=3 / 2$ is correct, see Secs. IV and $\mathrm{V}$.

\section{2. $1-C \gg \alpha$.}

Equation (B3) still applies as long as $\omega_{p 0}-\omega$ is positive and not too small. At larger frequencies, $\Pi(\omega)$ receives additional contribution from the pole $k_{*}$ of $D_{T}$ in the complex plane of $k$, which moves close to the real axis. The resonance linewidth $\Delta \omega_{p}$ is exponentially small,

$$
\Delta \omega_{p}=\frac{\operatorname{Im} \Pi\left(\omega_{p}\right)}{\rho \omega_{c}} \sim \omega_{p} e^{-F / 4 \alpha^{2}}
$$

where $F \simeq \Pi\left(\omega_{\text {th }}\right) /\left(C \Pi_{0}\right)-1$. The threshold frequency $\omega_{\text {th }}$ is only slightly below $\omega_{p}$, by an amount of the order of $\Delta \omega_{p}$. To be exact $\omega_{\text {th }}$ can be found from the condition that the solution of Eq. ( $\mathrm{B} 2$ ) is also the solution of the "derivative" of this equation with respect to $\Pi$,

$$
1=2 \mu C \Pi_{0} \int_{0}^{\infty} \frac{d k k\left[\epsilon \epsilon_{c}+(\Pi+Y k)^{2}\right]}{\left[\left(\Pi+\mu k^{2}\right)(\Pi+Y k)-\epsilon \epsilon_{c}\right]^{2}} .
$$

At frequencies somewhat higher than $\omega_{p}, \operatorname{Im} \Pi(\omega)$ is dominated by the aforementioned pole at $k_{*}=\left(\epsilon \epsilon_{c}-\Pi^{2}\right) / \Pi Y$ near the real axis, $\left|\operatorname{Re} k_{*}\right| \lessdot\left|\operatorname{Im} k_{*}\right|$. Im $\Pi$ is given simply by the residue of this pole 12

$$
\operatorname{Im} \Pi(\omega) \simeq 2 \pi \alpha^{2} C \Pi_{0}\left(\frac{\omega^{2}}{\omega_{p}^{2}}-1\right) .
$$

In conclusion, we would like to reiterate that neither of Eqs. (B3), (B4), or (B6) describes the dynamical response of the system correctly. We derived them here just to facilitate the comparison with the previous work on the subject. The correct expressions for $\operatorname{Im} \Pi$ and $\sigma_{x x}$ are given in Sec. IV.

${ }^{1}$ A. I. Larkin, Zh. Teor. Eksp. Fiz. 58, 1466 (1970) [Sov. Phys. JETP 31, 784 (1970)].

${ }^{2}$ G. Gruner, Rev. Mod. Phys. 60, 1129 (1988).

${ }^{3}$ Blatter et al., Rev. Mod. Phys. 66, 1125 (1994).

${ }^{4}$ J. Toner and D. P. DiVincenzo, Phys. Rev. B 41, 632 (1990).

${ }^{5}$ R. Seshadri and R. M. Westervelt, Phys. Rev. B 46, 5150 (1992).

${ }^{6}$ L. Radzihovsky and J. Toner, Phys. Rev. B 60, 206 (1999).

${ }^{7}$ Although crystallization of three-dimensional electrons has not yet been achieved, atomic ions [J. N. Tan, J. J. Bollinger, B. Jelenkovic, and D. J. Wineland, Phys. Rev. Lett. 75, 4198 (1995)], micron-size charged spheres in dusty plasmas [H. Thomas et al., Phys. Rev. Lett. 73, 
652 (1994)] and charged spheres in colloids [C. A. Murray et al., Phys. Rev. B 42, 688 (1990)] were demonstrated to assemble into $3 \mathrm{D}$ periodic structures.

${ }^{8}$ For review and references, see Chaps. 3 (by H. A. Fertig) and 9 (by M. Shayegan) in Perspectives in Quantum Hall Effect, edited by A. Piczuk and S. Das Sarma (Wiley, New York, 1997), and Two-Dimensional Electron Systems on Helium and Other Cryogenic Substrates, edited by E. Y. Andrei [Kluwer, Dordrecht (Netherlands), 1997].

${ }^{9}$ H. Fukuyama and P. A. Lee, Phys. Rev. B 17, 535 (1977).

${ }^{10}$ H. Fukuyama and P. A. Lee, Phys. Rev. B 18, 6245 (1978).

${ }^{11}$ L. Degiorgi and G. Grüner, Phys. Rev. B 44, 7820 (1991).

12 B. G. A. Normand, P. B. Littlewood, and A. J. Millis, Phys. Rev. B 46, 3920 (1992).

${ }^{13}$ M. A. Palaanen, R. L. Willett, P. B. Littlewood, R. R. Ruel, K. W. West, L. N. Pfeiffer, and D. J. Bishop, Phys. Rev. B 45, 11342 (1992).

${ }^{14}$ C.-C. Li, L. W. Engel, D. Shahar, D. C. Tsui, and M. Shayegan, Phys. Rev. Lett. 79, 1353 (1997).

${ }^{15}$ L. W. Engel, C.-C. Li, D. Shahar, D. C. Tsui, and M. Shayegan, Solid State Commun. 104, 167 (1997).

${ }^{16}$ C.-C. Li, J. Yoon, L. W. Engel, D. Shahar, D. C. Tsui, and M. Shayegan, cond-mat/9810066; C.-C. Li, Ph.D. thesis (Princeton University, 1999).

17 A. S. Beya, Ph.D. thesis (L'Université Paris 6, 1998).

${ }^{18}$ H. A. Fertig, Phys. Rev. B 59, 2120 (1999).

${ }^{19}$ R. Chitra, T. Giamarchi, and P. Le Doussal, Phys. Rev. Lett. 80, 3827 (1998)

${ }^{20}$ I. M. Lifshitz, S. A. Gredeskul, and L. A. Pastur, Introduction to The Theory of Disordered Systems (Wiley, New York, 1988).

21 T. Giamarchi, and P. Le Doussal, Phys. Rev. Lett. 72, 1530 (1994); Phys. Rev. B 52, 1242 (1995).

${ }^{22}$ L. Bonsall and A. A. Maradudin, Phys. Rev. B 15, 1959 (1977).

23 T. Natterman, S. Scheidl, S. E. Korshunov, and M. S. Li, J. Phys. I (France) 5, 565 (1995).

${ }^{24}$ C. Zeng, P. Leath, and D. S. Fisher, Phys. Rev. Lett. 82, 1935 (1999); C. Zeng, A. A. Middleton, and Y. Shapir, Phys. Rev. Lett. 77, 3204 (1996).

${ }^{25}$ D. Carpentier and P. Le Doussal, Phys. Rev. Lett. 81, 1881 (1998); ibid, 2558 (1998); cond-mat/9908335.

${ }^{26}$ T. Giamarchi and P. Le Doussal, cond-mat/9810218.

${ }^{27} W_{L, T}^{2}(r)$ should not be confused with quantities $B_{L, T}(r)$ introduced in Ref. 21. For $W_{L} \ll W_{T}, B_{L} \simeq B_{T} \simeq W_{T}^{2} / 2$.

${ }^{28}$ Incidentally, this means that in the $\omega \rightarrow 0$ limit, the collective-mode contribution to the magnetotransport has the same form as in the "Hall insulator," i.e., vanishing $\sigma_{x x}$ but classical $\rho_{x y}$. However, at finite $T$ and sufficiently low $\omega$ the single-particle contribution to the transport will become more important. Therefore, it would be unwarranted to draw the conclusion that the pinned WC behaves as a Hall insulator just on the basis of the phonon response studied here.

${ }^{29}$ A. F. Ioffe and A. R. Regel, Prog. Semicond. 4, 237 (1960); see also P. A. Lee and T. V. Ramakrishnan, Rev. Mod. Phys. 57, 287 (1985).

30 S. John, H. Somplolinsky, and M. J. Stephen, Phys. Rev. B 27, 5592 (1984); E. Akkermans and R. Manyard, Phys. Rev. B 32, 7850 (1985).

${ }^{31}$ N. N. Ablyazov, M. Yu. Kuchiev, and M. E. Raikh, Phys. Rev. B 44, 8802 (1991).

${ }^{32}$ P. Lloyd, J. Phys. C 2, 1717 (1969); see also C. Mudry, P. W. Brouwer, B. I. Halperin, V. Gurarie, and A. Zee, Phys. Rev. B 58, 13539 (1998).

${ }^{33}$ M. V. Feigelman, Zh. Eksp. Teor. Fiz. 79, 1095 (1980) [Sov. Phys. JETP 52, 555 (1980)]; V. M. Vinokur, M. B. Mineev, and M. V. Feigelman, Zh. Eksp. Teor. Fiz. 81, 2142 [Sov. Phys. JETP 54, 1138 (1981)]; M. V. Feigelman and V. M. Vinokur, Phys. Lett. 87A, 53 (1981).

${ }^{34}$ I. L. Aleiner and I. M. Ruzin, Phys. Rev. Lett. 72, 1056 (1994).

${ }^{35}$ H. Maurey and T. Giamarchi, Phys. Rev. B 51, 10833 (1995).

36 Yu. M. Galperin, V. G. Karpov, and V. I. Kozub, Adv. Phys. 38, 669 (1989).

37 P. K. Lam and S. M. Girvin, Phys. Rev. B 30, 473 (1980).

${ }^{38}$ Recently, H. Yi and H. A. Fertig [Phys. Rev. B 61, 5311 (2000)] studied a WC pinned by a commensurate pinning potential with a quartic anharmonicity $U(r)=\alpha r^{2}-\beta r^{4}$. The coefficients $\alpha$ and $\beta$ were taken to be identical for each lattice cite. In such a system the magnetophonon spectrum has a gap and the anharmonic broadening decays exponentially as $T \rightarrow 0$. In contrast, here we showed that in a truly random system where the phonon spectrum is gapless, the anharmonic effects are much stronger, persist even at zero temperature, and vary linearly with $T$.

${ }^{39}$ M. M. Fogler, unpublished.

${ }^{40}$ I. M. Ruzin, S. Marianer, and B. I. Shklovskii, Phys. Rev. B 46, 3999 (1992).

${ }^{41}$ K. Efetov, Supersymmetry in disorder and chaos (Cambridge University Press, New York, 1997).

${ }^{42}$ H. A. Fertig, private communication. 